\title{
Magnetic-conductivity effects on electromagnetic propagation in dispersive matter
}

\author{
Pedro D. S. Silva $\oplus^{*}$ Manoel M. Ferreira, Jr.๑, ${ }^{\dagger}$ and Marco Schreck $\odot^{\ddagger}$ \\ Departamento de Física, Universidade Federal do Maranhão, \\ Campus Universitário do Bacanga, São Luís (MA), 65080-805, Brazil \\ Luis F. Urrutia $\odot^{\S}$ \\ Instituto de Ciencias Nucleares, Universidad Nacional Autónoma de México, \\ 04510 México, Distrito Federal, Mexico
}

(Received 8 June 2020; accepted 31 August 2020; published 2 October 2020)

\begin{abstract}
The chiral magnetic effect (CME) has been investigated as a new transport phenomenon in condensed matter. Such an effect appears in systems with chiral fermions and involves an electric current generated by a magnetic field by means of an "exotic" magnetic conductivity. This effect can also be connected with extensions of the usual Ohm's law either in magnetohydrodynamics or in Lorentz-violating scenarios. In this work, we study the classical propagation of electromagnetic waves in isotropic dispersive matter subject to a generalized Ohm's law. The latter involves currents linear in the magnetic field and implies scenarios inducing parity violation. We pay special attention to the case of a vanishing electric conductivity. For a diagonal magnetic conductivity, which includes the CME, the refractive index is modified such that it leads to birefringence. For a nondiagonal magnetic conductivity, modified refractive indices exhibiting imaginary parts occur ascribing a conducting behavior to a usual dielectric medium. Our findings provide new insights into typical material properties associated with a magnetic conductivity.
\end{abstract}

DOI: 10.1103/PhysRevD.102.076001

\section{INTRODUCTION}

The chiral magnetic effect (CME) is the macroscopic generation of an electric current in the presence of a magnetic field as the result of an asymmetry between the number density of left- and right-handed chiral fermions. It leads to a current that is linear in the magnetic field [1]. This quantum effect has been the subject of extensive research in particle and field theory as well as nuclear and condensed matter physics. It was investigated in quark-gluon plasmas with a chiral chemical potential under the influence of an external magnetic field $[2,3]$ and was also derived in the context of high-energy physics by Vilenkin (in the 1980s) [4,5] who supposed an imbalance of fermion chirality in the presence of cosmic magnetic fields in the early Universe. The CME was studied in cosmology [6], as well, where it was applied to explain the origin of the

\footnotetext{
pedro.dss@discente.ufma.br

manojr.ufma@gmail.com

*marco.schreck@ufma.br

§urrutia@nucleares.unam.mx
}

Published by the American Physical Society under the terms of the Creative Commons Attribution 4.0 International license. Further distribution of this work must maintain attribution to the author(s) and the published article's title, journal citation, and DOI. Funded by SCOAP ${ }^{3}$. very high magnetic field strengths (up to $10^{15} \mathrm{G}$ ) observed in neutron stars $[7,8]$. An interesting question concerns the possible impact of the external axial-vector field $V_{5}^{\mu}$ on the magnitude of the anomalous current in the CME [9], which was also examined for the polarization tensor of a photon in a fermion plasma under the influence of $V_{5}^{\mu}$ [10]. Connections between the $\mathrm{CME}$ and matter subject to the electroweak interaction were established, too $[11,12]$.

In condensed matter systems, the CME plays a most important role. It appears as a relevant effect in Weyl semimetals, where it is usually connected to the chiral anomaly associated with Weyl nodal points [13]. In such materials, massless fermions acquire a drift velocity along the magnetic field, whose direction is given by their chirality. Opposite chirality implies opposite velocities, creating a chiral-fermion imbalance that is proportional to the chiral magnetic current. The first experimental observation of the CME was reported in 2014 [14]. Several investigations have been carried out on the properties of this phenomenon, including the CME in the absence of Weyl nodes [15], anisotropic effects stemming from tilted Weyl cones [16], the CME and anomalous transport in Weyl semimetals [17], quantum oscillations arising from the CME [18], computation of the electromagnetic fields produced by an electric charge near a topological Weyl semimetal with two Weyl nodes [19], and chiral superconductivity [20]. 
An interesting relation between the CME and MaxwellCarroll-Field-Jackiw electrodynamics has been established in the literature [21] by examining the connection between the CME and Lorentz-violating theories including axion electrodynamics. The possibility of Lorentz invariance violation was proposed in the context of physics at the Planck scale such as strings [22]. Presently, the Standard Model extension [23], where fixed background tensor fields are coupled to the dynamical fields, is usually employed to parametrize it. A violation in the photon sector can occur by means of a $C P T$-odd [24] or $C P T$-even term [25]. A Lorentz-violating extension for the current density that resembles the macroscopic description of the CME can be found in Ref. [26], where some preliminary studies of aspects of Lorentz-violating electrodynamics in continuous matter were performed.

Additional motivation for getting more insight into conduction currents driven by magnetic fields comes from magnetohydrodynamics. After making some simplifying assumptions for plasmas of colliding particles, the resistive Ohm's law for the one-fluid model is written as [27]

$$
(\mathbf{E}+\mathbf{V} \times \mathbf{B})^{i}=\eta^{i j} J^{j} .
$$

Here $\mathbf{V}$ is the average velocity of the electrons and ions, while $\mathbf{J}$ is the total current density. The expression $\eta^{i j} J^{j}$ corresponds to an effective collision term for electrons and ions and the resistivity $\eta^{i j}$ can, in general, be a tensor. Even if Eq. (1) is not a rigorous model, it is widely used, because it captures the most important deviations from the ideal magnetohydrodynamic model. Inverting Eq. (1) yields $J^{i}=\sigma_{i j} E^{j}+\sigma_{i j}^{B} B^{j}$, with $\sigma_{i j}$ being the inverse of $\eta^{i j}$ and $\sigma_{i j}^{B}=\sigma_{i p} V_{q} \epsilon_{p q j}$ (where $\epsilon_{i j k}$ is the three-dimensional LeviCivita symbol) defining a magnetic conductivity. In the following, we will generalize this motivation by considering the magnetic conductivity tensor $\sigma_{i j}^{B}$ to be completely independent of the electric conductivity tensor $\sigma_{i j}$.

The main purpose of this work is to analyze the possible phenomena of a magnetic conductivity in a usual dielectric medium. In particular, we are interested in investigating the effects originating from a magnetic conductivity on the propagation of electromagnetic waves in a dispersive dielectric continuous medium characterized by the parameters $\epsilon$ (electric permittivity), $\mu$ (magnetic permeability), and $\sigma$ (Ohmic conductivity). In this sense, we propose some particular configurations for the magnetic conductivity to be explored.

We start from the Maxwell equations in a continuous medium, supplemented by the constitutive relations $\mathbf{D}=\epsilon \mathbf{E}$, $\mathbf{B}=\mu \mathbf{H}$, and the magnetic current, $J_{\mathrm{CME}}^{i}=\sigma_{i j}^{B} B^{j}$, arising from an extension of Ohm's law. We obtain the corresponding refractive indices and electric fields for the propagating modes in some scenarios. First, we address an isotropic and an anisotropic diagonal conductivity tensor, configurations which describe the chiral magnetic current observed in Weyl semimetals. After doing so, we examine more exotic configurations of antisymmetric and symmetric nondiagonal conductivity tensors. In what follows, we will take $\epsilon, \mu, \sigma \in \mathbb{R}$.

On the one hand, the isotropic or anisotropic diagonal magnetic conductivity tensors generate scenarios of birefringent dielectric crystals, described by two different refractive indices for each wave vector. On the other hand, the nondiagonal anisotropic magnetic conductivity (symmetric or antisymmetric) creates the remarkable behavior of a conducting phase in the dielectric substrate.

This work is outlined as follows. In Sec. II we briefly review some basic aspects of electrodynamics in matter, focusing on the refractive index, constitutive relations, the generalized form of Ohm's law, and the magnetic current. In Sec. III we discuss the general effect of a magnetic conductivity on wave propagation in continuous dielectric matter. We analyze diagonal conductivity tensors and some special cases of an exotic conductivity. Section IV is dedicated to deriving the contributions to the charge and current density that result from the magnetic conductivity and are needed to guarantee the consistency of Maxwell's equations. Furthermore, in Sec. V we study what impact a magnetic conductivity has on certain phenomena such as the skin effect and reflection. Finally, we summarize our results in Sec. VI.

\section{BASIC ASPECTS OF ELECTRODYNAMICS IN MATTER}

The electrodynamic properties of a continuous medium are characterized by its electric permittivity $\epsilon$, magnetic permeability $\mu$, and Ohmic conductivity $\sigma$. The static and dynamic behavior is described by the standard Maxwell equations,

$$
\begin{array}{ll}
\nabla \cdot \mathbf{D}=\rho, & \nabla \times \mathbf{H}-\partial_{0} \mathbf{D}=\mathbf{J}, \\
\nabla \cdot \mathbf{B}=0, & \nabla \times \mathbf{E}+\partial_{0} \mathbf{B}=\mathbf{0} .
\end{array}
$$

The response of the medium to applied electromagnetic fields is measured in terms of the polarization vector $\mathbf{P}$ and magnetization vector $\mathbf{M}$, defined by linear constitutive relations: $\mathbf{P}=\epsilon_{0} \chi_{E} \mathbf{E}$ and $\mathbf{M}=\chi_{M} \mathbf{H}$, where $\chi_{E}$ and $\chi_{M}$ are the electric and magnetic susceptibility, respectively, of an isotropic ponderable medium. Such relations allow us to define the electric displacement field $\mathbf{D}$ and the magnetic flux density $\mathbf{B}$ :

$$
\begin{gathered}
\mathbf{D}=\epsilon_{0} \mathbf{E}+\mathbf{P}=\epsilon_{0}\left(1+\chi_{E}\right) \mathbf{E}=\epsilon \mathbf{E}, \\
\mathbf{B}=\mu_{0} \mathbf{H}+\mu_{0} \mathbf{M}=\mu_{0}\left(1+\chi_{M}\right) \mathbf{H}=\mu \mathbf{H} .
\end{gathered}
$$

We then rewrite Eqs. (3a) and (3b) as 


$$
\left(\begin{array}{l}
\mathbf{D} \\
\mathbf{H}
\end{array}\right)=\left(\begin{array}{cc}
\epsilon \mathbb{1} & 0 \\
0 & \mu^{-1} \mathbb{1}
\end{array}\right)\left(\begin{array}{l}
\mathbf{E} \\
\mathbf{B}
\end{array}\right)
$$

with the identity matrix $\mathbb{1}$ in three spatial dimensions. By using a plane-wave ansatz for the electromagnetic fields, $\mathbf{E}=\mathbf{E}_{0} e^{\mathrm{i}(\mathbf{k} \cdot \mathbf{r}-\omega t)}$ and $\mathbf{B}=\mathbf{B}_{0} e^{\mathrm{i}(\mathbf{k} \cdot \mathbf{r}-\omega t)}$, the Maxwell equations and $\mathbf{J}=\sigma \mathbf{E}$ yield

$$
\mathbf{k} \times \mathbf{k} \times \mathbf{E}+\omega^{2} \mu \bar{\epsilon}(\omega) \mathbf{E}=0,
$$

with $\mathbf{k}^{2}=\omega^{2} \mu \bar{\epsilon}(\omega)$, where

$$
\bar{\epsilon}(\omega)=\epsilon+\mathrm{i} \frac{\sigma}{\omega},
$$

is the frequency-dependent electric permittivity of the medium. This complex permittivity leads to a complex refractive index: ${ }^{1}$

$$
\bar{n}=+\frac{\sqrt{\mathbf{k}^{2}}}{\omega}=\sqrt{\mu \epsilon+\mathrm{i} \frac{\mu \sigma}{\omega}}=n^{\prime}+\mathrm{i} n^{\prime \prime},
$$

where

$$
n^{\prime \prime \prime \prime}=\sqrt{\sqrt{\Upsilon_{0}^{2}+\left(\frac{\mu \sigma}{2 \omega}\right)^{2}} \pm \Upsilon_{0},} \quad \Upsilon_{0}=\frac{\mu \epsilon}{2} .
$$

The imaginary part of the refractive index implies a real exponential factor, $e^{-\omega n^{\prime \prime}(\hat{\mathbf{k}} \cdot \mathbf{r})}$ with $\hat{\mathbf{k}} \equiv \mathbf{k} / \sqrt{\mathbf{k}^{2}}$, in the plane-wave solutions for the electromagnetic fields. This term damps the amplitude of the wave along its propagation through matter and is related to the absorption coefficient $\alpha=2 \omega n^{\prime \prime}$, whose inverse value determines the penetration depth. Such a scenario is typical for a conducting medium.

In a linear, continuous, ponderable medium, general constitutive relations can be envisaged as a theoretical possibility [28], which gained great attention with the advent of topological insulators [29,30]. In systems of this kind, it holds that

$$
\left(\begin{array}{l}
\mathbf{D} \\
\mathbf{H}
\end{array}\right)=\left(\begin{array}{cc}
\epsilon \mathbb{1} & \alpha \mathbb{1} \\
\beta \mathbb{1} & \mu^{-1} \mathbb{1}
\end{array}\right)\left(\begin{array}{l}
\mathbf{E} \\
\mathbf{B}
\end{array}\right),
$$

with additional material parameters $\alpha, \beta$ that are not independent and whose sum provides what is known as the "activity constant" of a medium. Such an extension also

\footnotetext{
${ }^{1}$ Note that the refractive index can be a complex function, in general. Thus, instead of employing the norm $|\mathbf{k}|$ in the definition of the refractive index, which is a non-negative, real number, we use $+\sqrt{\mathbf{k}^{2}}$. Furthermore, we only consider refractive indices with a non-negative real part, which is indicated explicitly by the plus sign in front of the square root.
}

occurs in a Lorentz-violating anisotropic electrodynamics [26] where

$$
\left(\begin{array}{l}
\mathbf{D} \\
\mathbf{H}
\end{array}\right)=\left(\begin{array}{cc}
\epsilon \mathbb{1}+\kappa_{D E} & \kappa_{D B} \\
\kappa_{H E} & \mu^{-1} \mathbb{1}+\kappa_{H B}
\end{array}\right)\left(\begin{array}{l}
\mathbf{E} \\
\mathbf{B}
\end{array}\right),
$$

with dimensionless $(3 \times 3)$ matrices $\kappa_{D E}, \kappa_{D B}, \kappa_{H E}$, and $\kappa_{H B}$ that are composed of a vacuum part and a matter part. In component form, the latter relations read

$$
\begin{gathered}
D^{i}=\left[\epsilon \delta_{i j}+\left(\kappa_{D E}\right)_{i j}\right] E^{j}+\left(\kappa_{D B}\right)_{i j} B^{j}, \\
H^{i}=\left[\mu^{-1} \delta_{i j}+\left(\kappa_{H B}\right)_{i j}\right] B^{j}+\left(\kappa_{H E}\right)_{i j} E^{j}
\end{gathered}
$$

These generalized scenarios lead to an unusual electrodynamics where the electric displacement field receives a contribution from the magnetic flux density and the magnetic field gets a contribution from the electric field. Such modified constitutive relations, $\mathbf{D}=\mathbf{D}(\mathbf{E}, \mathbf{B})$ and $\mathbf{H}=\mathbf{H}(\mathbf{E}, \mathbf{B})$ are observed, for example, for topological insulators [29].

At this point, we introduce a generalized Ohm's law for the current density $\mathbf{J}$, considering the contribution of the magnetic current, $\mathbf{J}_{\mathrm{CME}}=\sigma^{B} \cdot \mathbf{B}$, that is,

$$
J^{i}=\sigma_{i j} E^{j}+\sigma_{i j}^{B} B^{j},
$$

composed of the usual Ohmic term involving the conductivity tensor $\sigma_{i j}$ as well as an exotic term with the magnetic conductivity tensor $\sigma_{i j}^{B}$, which has found realization in some condensed matter systems. The second term can also be proposed as an extension of Ohm's law in magnetohydrodynamics [27] (see also Sec. I) as well as in Lorentzviolating scenarios [26].

The magnetic conductivity tensor, $\sigma_{i j}^{B}$, is even under time reversal $(T)$ and charge conjugation $(C)$, but odd under parity transformations $(P)$; see Table I. Most notably, this tensor is even under time reversal, since $\mathbf{J}$ and $\mathbf{B}$ are $T$ odd, which is highly unusual for a conductivity. This behavior is analog to that of the phenomenological parameter $\mu$ (not to be confused with the magnetic permeability) observed in London's superconductivity model $\left(\mathbf{J}=-\mu^{2} \mathbf{A}\right)$ [20]. The $T$-even character of $\sigma_{i j}^{B}$ is typical of nondissipative and reversible processes $[1,20]$. This property is what distinguishes the magnetic conductivity from the usual Ohmic conductivity, which is $T$ odd

TABLE I. Behavior of the Ohmic and exotic conductivity, respectively, under $C, P$, and $T$ transformations.

\begin{tabular}{llllll}
\hline \hline & $\mathbf{E}$ & $\mathbf{B}$ & $\mathbf{J}$ & $\sigma$ & $\sigma^{B}$ \\
\hline$C$ & - & - & - & + & + \\
$P$ & - & + & - & + & - \\
$T$ & + & - & - & - & + \\
\hline \hline
\end{tabular}


and compatible with dissipative and nonreversible phenomena. Table I shows a comparative analysis between the magnetic and Ohmic conductivity tensors under discrete symmetry transformations, revealing crucial differences when the former is subject to $T, P, C P$, and $C T$ transformations.

Using the generalized Ohm's law of Eq. (11) and conventional isotropic constitutive relations, $D^{i}=\epsilon \delta_{i j} E^{j}$, $H^{i}=\mu^{-1} \delta_{i j} B^{j}$, in the Maxwell equations, Eq. (5) keeps its general form:

$$
[\mathbf{k} \times \mathbf{k} \times \mathbf{E}]^{i}+\omega^{2} \mu \bar{\epsilon}_{i j}(\omega) E^{j}=0,
$$

where

$$
\bar{\epsilon}_{i j}(\omega)=\left(\epsilon+\mathrm{i} \frac{\sigma}{\omega}\right) \delta_{i j}+\frac{\mathrm{i}}{\omega^{2}}\left(\sigma^{B}\right)_{i a} \epsilon_{a b j} k_{b},
$$

defines the frequency-dependent extended permittivity tensor (EPT). Equation (12a) implies

$$
\left[\mathbf{k}^{2} \delta_{i j}-k_{i} k_{j}-\omega^{2} \mu \bar{\epsilon}_{i j}\right] E^{j}=0 .
$$

Notice that the latter equation yields $k_{i} \bar{\epsilon}_{i j} E^{j}=0$, where we can interpret $\bar{D}^{i}=\bar{\epsilon}_{i j} E^{j}$ as an extended displacement vector.

For a general anisotropic continuous scenario, we write $\mathbf{k}=\omega \mathbf{n}$ where $\mathbf{n}$ is a vector pointing along the direction of the wave vector and yielding the refractive index: ${ }^{2}$ $n=+\sqrt{\mathbf{n}^{2}}$. Hence, Eq. (13) becomes

$$
\left[n^{2} \delta_{i j}-n^{i} n^{j}-\mu \bar{\epsilon}_{i j}\right] E^{j}=0 .
$$

The latter can also be cast into the form

$$
M_{i j} E^{j}=0,
$$

where the tensor $M_{i j}$ reads

$$
M_{i j}=n^{2} \delta_{i j}-n_{i} n_{j}-\mu \bar{\epsilon}_{i j},
$$

and $\bar{\epsilon}_{i j}$ is given by Eq. (12b). The set of equations given above has nontrivial solutions for the electric field if the determinant of the coefficient matrix $M_{i j}$ vanishes. This condition on the determinant provides the dispersion relations associated with wave propagation in the medium.

In the following, we will discard refractive indices with negative real parts associated with frequencies that have the same property. Sophisticated composites of different materials can be designed that have negative permittivity and

\footnotetext{
${ }^{2}$ Here we again take into account that the norm $|\mathbf{n}|$ is nonnegative. To permit complex refractive indices, we consider $+\sqrt{\mathbf{n}^{2}}$ instead of $|\mathbf{n}|$. The plus sign indicates that we discard refractive indices with negative real parts.
}

permeability. These are called metamaterials [31] and the real parts of their refractive indices must be endowed with \pm signs according to the materials at the interface: material-material $(+)$, material-metamaterial $(-)$, or metamaterial-metamaterial $(+)$. On the contrary, negative refractive indices are not known to occur in crystals found in nature, which is our focus in this paper.

\section{PROPAGATION BEHAVIOR UNDER CHIRAL AND EXOTIC MAGNETIC CONDUCTIVITY}

In this section, we will investigate the effects stemming from chiral as well as exotic magnetic conductivities, incorporated into the formalism used for describing the propagation of electromagnetic waves in anisotropic dispersive media. In order to examine the magnetic current $J_{\mathrm{CME}}^{i}=\sigma_{i j}^{B} B^{j}$ classically, we consider the conductivity tensor $\sigma_{i j}^{B}$ in Eqs. (12b) and (15) originating from the emergence of a magnetic field. Studies of the CME $[1,2,20,21]$ have reported the generation of an electric current induced by a magnetic field,

$$
J_{\mathrm{CME}}^{i}=\frac{e^{2}}{4 \pi^{2}}(\Delta \mu) B^{i} \equiv \Sigma B^{i},
$$

where $e$ is the fermion charge, $\mathbf{B}$ the applied magnetic flux density, $\Delta \mu \equiv \mu_{R}-\mu_{L}$ is also known as the chiral chemical potential, and $\Sigma$ is the chiral magnetic conductivity [6-13]. As written in Eq. (16), this effect is clearly represented by an isotropic diagonal magnetic conductivity, that is,

$$
\sigma_{i j}^{B}=\Sigma \delta_{i j}
$$

in which

$$
\Sigma=\frac{e^{2}}{4 \pi^{2}} \Delta \mu .
$$

We can write down the magnetic conductivity $\sigma_{i j}^{B}$ in the following form:

$$
\sigma_{i j}^{B}=\Sigma \delta_{i j}+\Sigma_{i j},
$$

where $\Sigma$ is $1 / 3$ of the trace of the $\sigma_{i j}^{B}$ matrix and represents the isotropic part of this conductivity, while $\Sigma_{i j}$ stands for all off-diagonal components of $\sigma_{i j}^{B}$. Hence, the diagonal piece of the conductivity tensor is related to the CME and constitutes the first case to be analyzed. We further examine the generalization of the magnetic conductivity to exotic scenarios,

$$
J^{i}=\Sigma_{i j} B^{j},
$$

where $\Sigma_{i j}$ comprises off-diagonal or anisotropic conductivity components. It is worthwhile to note that the anisotropic chiral magnetic effect [16] represents an 
interesting theoretical possibility to be proposed and investigated. At first, it can be induced by a diagonal anisotropic conductivity tensor, as will be examined in Sec. III B.

\section{A. Isotropic diagonal chiral conductivity}

First of all, we discuss the behavior of an isotropic magnetic conductivity, which is represented by a diagonal matrix; cf. Eq. (17a). By inserting the latter into Eq. (12b), one obtains

$$
\bar{\epsilon}_{i j}(\omega)=\left(\epsilon+\mathrm{i} \frac{\sigma}{\omega}\right) \delta_{i j}-\frac{\mathrm{i} \Sigma}{\omega^{2}} \epsilon_{i j b} k_{b},
$$

where the last term of Eq. (20) represents the contribution from the chiral conductivity. Note that we are starting from an isotropic permittivity tensor, $\epsilon \delta_{i j}$, where all effects that are usually related to anisotropies in media manifest themselves via the way the magnetic conductivity is coupled to the fields. In this case, the tensor $M_{i j}$ given by Eq. (15b), has the form

$$
\left[M_{i j}\right]=\left(\begin{array}{ccc}
n_{2}^{2}+n_{3}^{2}-\mu \epsilon-\mathrm{i} \mu \frac{\sigma}{\omega} & -n_{1} n_{2}+\mathrm{i} \mu \frac{n_{3} \Sigma}{\omega} & -n_{1} n_{3}-\mathrm{i} \mu \frac{n_{2} \Sigma}{\omega} \\
-n_{1} n_{2}-\mathrm{i} \mu \frac{n_{3} \Sigma}{\omega} & n_{1}^{2}+n_{3}^{2}-\mu \epsilon-\mathrm{i} \mu \frac{\sigma}{\omega} & -n_{2} n_{3}+\mathrm{i} \mu \frac{n_{1} \Sigma}{\omega} \\
-n_{1} n_{3}+\mathrm{i} \mu \frac{n_{2} \Sigma}{\omega} & -n_{2} n_{3}-\mathrm{i} \mu \frac{n_{1} \Sigma}{\omega} & n_{1}^{2}+n_{2}^{2}-\mu \epsilon-\mathrm{i} \mu \frac{\sigma}{\omega}
\end{array}\right)
$$

Requiring $\operatorname{det}\left[M_{i j}\right]=0$, we get

$$
\begin{gathered}
n_{ \pm}^{2}=4 \Upsilon_{\Sigma}+\mu\left(-\epsilon+\mathrm{i} \frac{\sigma}{\omega}\right) \pm \frac{\mu \Sigma}{\omega} \sqrt{2 \Upsilon_{\Sigma}+\mathrm{i} \mu \frac{\sigma}{\omega}}, \\
2 \Upsilon_{\Sigma}=\mu \epsilon+\left(\frac{\mu}{2 \omega} \Sigma\right)^{2}
\end{gathered}
$$

which can be split into real and imaginary parts as follows:

$$
n_{ \pm}^{2}=\mu \epsilon+\frac{\mu \Sigma}{\omega}\left(\frac{\mu \Sigma}{2 \omega} \pm N_{+}\right)+\mathrm{i} \frac{\mu}{\omega}\left(\sigma \pm \Sigma N_{-}\right),
$$

where

$$
N_{ \pm}=\sqrt{\sqrt{\Upsilon_{\Sigma}^{2}+\left(\frac{\mu \sigma}{2 \omega}\right)^{2}} \pm \Upsilon_{\Sigma}}
$$

Equation (23a) yields two distinct refractive indices for each frequency $\omega$, which is compatible with the physics of a conducting dielectric medium endowed with birefringence. This behavior already occurs for the isotropic conductivity tensor of Eq. (17a), revealing that birefringence comes from the way the chiral conductivity is coupled to the fields. Thus, when considered within a medium of Ohmic conductivity $(\sigma \neq 0)$, a chiral conductivity modifies the refractive index of the conducting medium. It alters the phase velocity associated with its real part and the absorption (or attenuation) coefficient related to its imaginary part.

\section{Dielectric nonconducting medium}

In the case where we start from a dielectric medium with zero Ohmic conductivity, $\sigma=0$, Eq. (22a) provides two distinct real values for the refractive index,

$$
n_{ \pm}^{2}=4 \Upsilon_{\Sigma}-\mu \epsilon \pm \frac{\mu \Sigma}{\omega} \sqrt{2 \Upsilon_{\Sigma}}
$$

which characterizes a dispersive nonconducting behavior typical of a uniaxial crystal, where the propagation occurs with different velocities along the principal dielectric directions [32]. Therefore, the system will behave like a birefringent dispersive dielectric medium where electromagnetic waves propagate without undergoing attenuation (nonconducting or absorbing behavior). The corresponding refractive indices are given by

$$
n_{ \pm}=\sqrt{2 \Upsilon_{\Sigma}} \pm \frac{\mu \Sigma}{2 \omega}
$$

with $\Upsilon_{\Sigma}$ of Eq. (22b). The square of the latter leads back to Eq. (24). In the present configuration, it is important to point out that the chiral conductivity implies a typical conducting behavior for the medium only when it is defined simultaneously with the Ohmic conductivity $\left(\sigma \neq 0, \sigma^{B} \neq 0\right)$, as shown in the complex refractive index of Eq. (23a). When it is defined for a nonconducting dielectric $\left(\sigma=0, \sigma^{B} \neq 0\right)$, the behavior remains that of a dispersive nonabsorbing medium, as indicated by the complex refractive index in Eq. (24). This is because $N_{-}=0$ when $\sigma=0$.

Alternatively, a refractive index can be determined from the frequency as a function of the wave vector, $\omega=\omega(\mathbf{k})$, via the definition $n \equiv+\sqrt{\mathbf{k}^{2}} / \omega(\mathbf{k})$ [see Eq. (7a)]. The possible frequencies $\omega$ also follow from the requirement that $\operatorname{det}\left[M_{i j}\right]=0$ and are associated with particular modes of the electric field. In order to better examine the features of propagation, we implement $n=\sqrt{\mathbf{k}^{2}} / \omega$ in Eq. (24) and obtain the dispersion equation 


$$
\omega^{4}-2 \omega^{2} \frac{k^{2}}{\mu \epsilon}+\left(\frac{k^{2}}{\mu \epsilon}-\frac{\mu \Sigma^{2}}{2 \epsilon}\right)^{2}-\frac{\mu^{2} \Sigma^{4}}{4 \epsilon^{2}}=0,
$$

with $k \equiv \sqrt{\mathbf{k}^{2}}$. Solving for $\omega$, one gets

$$
\omega_{ \pm}^{2}=\frac{k^{2}}{\mu \epsilon}\left(1 \pm \frac{\mu \Sigma}{k}\right)
$$

Equation (27) represents two distinct modes, $\omega_{+}$and $\omega_{-}$. While the frequency $\omega_{+}$is real for any value of $k$, the frequency $\omega_{-}$of the second mode can be imaginary if $k<\Sigma \mu$. To ensure that $\omega_{-}$represents the frequency of a physical propagating mode, we should require that $k>\Sigma \mu$. Birefringence occurs when distinct polarization modes propagate with different phase velocities. In this medium of zero Ohmic conductivity, the phase velocities are

$$
v_{\mathrm{ph}( \pm)}=\frac{\omega_{ \pm}}{k}=\frac{1}{\sqrt{\mu \epsilon}} \sqrt{1 \pm \frac{\mu \Sigma}{k}},
$$

yielding the following phase velocity difference:

$$
\begin{aligned}
\Delta v_{\mathrm{ph}} & =\frac{1}{\sqrt{\mu \epsilon}}\left[\sqrt{1+\frac{\mu \Sigma}{k}}-\sqrt{1-\frac{\mu \Sigma}{k}}\right] \\
& \simeq \frac{1}{\sqrt{\mu \epsilon}} \frac{\mu \Sigma}{k}
\end{aligned}
$$

showing that the trace $\Sigma$ of the isotropic chiral conductivity is really responsible for birefringence. Thus, a diagonal isotropic $\sigma_{i j}^{B}$ generates a birefringent dispersive nonconducting behavior.

We can also analyze the effect that such a term has on the group velocity,

$$
v_{g( \pm)}=\left|\frac{\partial \omega_{ \pm}}{\partial \mathbf{k}}\right|=\frac{1}{\sqrt{\mu \epsilon}} \frac{1 \pm \mu \Sigma /(2 k)}{\sqrt{1 \pm \mu \Sigma / k}},
$$

which has a singularity for small momenta indicating problems with classical causality due to $v_{g( \pm)}>1$. Causality is preserved for wave propagation in the largemomentum regime.

One can also obtain the refractive indices for this medium $(\sigma=0)$ by diagonalizing the permittivity tensor of Eq. (20) and setting each eigenvalue equal to $n^{2} / \mu$. In general, this procedure provides the refractive indices only for the propagation along the directions of the principal axes of $\bar{\epsilon}_{i j}$.

The eigenvalues $\epsilon_{a}(a=1,2,3)$, where $\bar{\epsilon}_{i j} e_{a}^{j}=\epsilon_{a} e_{a}^{i}$ (with eigenvectors $\mathbf{e}_{a}$ ), are given by

$$
\begin{gathered}
\epsilon_{1}=\epsilon, \\
\epsilon_{2,3} \equiv \epsilon_{ \pm}=\epsilon \pm \frac{\Sigma}{\omega} n,
\end{gathered}
$$

which can be associated with the following refractive indices:

$$
\begin{aligned}
& n^{2}=\mu \epsilon, \\
& n_{ \pm}^{2}=\mu \epsilon \pm \frac{\mu \Sigma}{\omega} n_{ \pm} .
\end{aligned}
$$

Surprisingly, the latter result reproduces Eq. (25), stemming from $\operatorname{det}\left[M_{i j}\right]=0$ and valid for an arbitrary direction, which means that the eigenvalues $\epsilon_{2}$ and $\epsilon_{3}$ correspond to the refractive indices of the medium $n_{+}$and $n_{-}$, respectively.

In the following, we explain this behavior. The proposed method of finding the refractive indices $n$ through the equation $n^{2}=\mu \epsilon_{a}(n)$, where $\epsilon_{a}$ are the eigenvalues of the EPT $\bar{\epsilon}_{i j}$, only works under the requirement described below. For a general vector $\mathbf{n}$, the related electric field $\mathbf{E}_{a}$, which satisfies the condition $M_{i j} E_{a}^{j}=0$ according to Eq. (14), must be such that $\mathbf{n} \cdot \mathbf{E}_{a}=0$. In this case, diagonalizing $M$ is equivalent to diagonalizing $\bar{\epsilon}$ and the result $\mathbf{E}_{a} \sim \mathbf{e}_{a}$ follows. Notice that we must also have $\mathbf{n} \cdot \mathbf{e}_{a}=0$. This situation is clearly illustrated in the present case, where Eqs. (14) and (20) yield the general condition $\mathbf{n} \cdot \mathbf{E}=0$. Here, the three eigenvectors of the generalized permittivity are

$$
\begin{aligned}
\mathbf{e}_{1} & =\frac{\mathbf{n}}{n} \equiv \mathbf{m}, \\
\mathbf{e}_{2,3} & =\frac{1}{\sqrt{2\left(m_{1}^{2}+m_{3}^{2}\right)}}\left(\begin{array}{c}
m_{3} \mp \mathrm{i} m_{1} m_{2} \\
\pm \mathrm{i}\left(m_{1}^{2}+m_{3}^{2}\right) \\
\mp \mathrm{i} m_{2} m_{3}-m_{1}
\end{array}\right),
\end{aligned}
$$

with the unit vector $\mathbf{m}$ defining the planes of constant phase of the wave. Let us observe that the eigenvectors in Eqs. (33a) and (33b) are independent of the corresponding refractive indices $n_{ \pm}$, being just functions of the direction given by $\mathbf{m}$. We note that $\mathbf{e}_{1} \cdot \mathbf{e}_{2}^{*}=\mathbf{e}_{1} \cdot \mathbf{e}_{3}^{*}=\mathbf{e}_{2} \cdot \mathbf{e}_{3}^{*}=0$, whereupon these three eigenvectors are linearly independent. In particular, $\mathbf{e}_{2}$ and $\mathbf{e}_{3}$ are orthogonal to $\mathbf{e}_{1}=\mathbf{m}$, thus yielding the correct refractive indices $n_{ \pm}$of Eq. (24), according to the proposed method. In this case, the propagating modes of the electric field are correctly described by the eigenvectors $\mathbf{e}_{2}$ and $\mathbf{e}_{3}$ and the eigenvalue $\epsilon_{1}=\epsilon$ has to be rejected, because $\mathbf{m} \cdot \mathbf{e}_{1}$ is nonzero.

To decide which refractive indices are physical, we can also look at the modes of the electric field. The latter are obtained from solving the homogeneous system of equations $M_{i j} E^{j}=0$ for $\mathbf{E}$ with $\omega$ replaced by the dispersion relations $\omega(\mathbf{k})$ determined from the coefficient determinant. In the dielectric nonconducting medium under consideration, a particular frequency does not correspond to a physical mode when the electric field is longitudinal. In other words, a mode is unphysical when its electric field 
points along the direction of the wave vector, i.e., $\mathbf{E} \cdot \mathbf{k}=$ $|\mathbf{E}||\mathbf{k}|$ or $\mathbf{k} \times \mathbf{E}=\mathbf{0}$. As $[M, \bar{\epsilon}]=0$ for the particular isotropic configuration of Eq. (17a), the eigenvector of Eq. (33a) also corresponds to a mode of the electric field. As it is longitudinal, the associated solution for the permittivity $\epsilon_{1}=\epsilon$ cannot be physical and must be discarded.

Since the configuration studied is isotropic, we can choose $\mathbf{m}=(0,0, m)$ without loss of generality. Equation (33b) then results in

$$
\mathbf{e}_{2,3}=\frac{1}{\sqrt{2}}\left(\begin{array}{c}
1 \\
\pm \mathrm{i} \\
0
\end{array}\right) .
$$

The vectors $\mathbf{e}_{2}$ and $\mathbf{e}_{3}$, respectively, can be interpreted as the polarization vectors of left-handed $(L)$ and righthanded $(R)$ polarized electromagnetic waves. ${ }^{3}$ Hence, we identify $n_{L, R} \equiv n_{ \pm}$.

These polarizations are transverse, i.e., perpendicular to m. According to Table I, the magnetic conductivity $\sigma_{i j}^{B}$ is odd under parity transformations. While parity violation does not show up in a single refractive index of Eq. (25) on its own, it becomes manifest in the distinct propagation properties of left- and right-handed polarized electromagnetic waves. This is the physical reason for birefringence.

The behavior found for this configuration is highly interesting. Parity violation could be expected to imply refractive indices that are angular dependent. In other words, birefringence in a material is usually caused by the presence of at least a single optical axis. An optical axis indicates a preferred direction in the crystal, whereupon its refractive index cannot be isotropic, anymore. Hence, under usual circumstances, an occurrence of parity violation and birefringence seems to contradict isotropy of a crystal. If a magnetic conductivity is present, we found that birefringence can also emerge in an isotropic crystal.

If birefringence occurs in a medium with a single optical axis, a light ray can split into an ordinary and an extraordinary ray. The ordinary one behaves according to Snell's law whereas the extraordinary one does not do so. The electric field associated with the extraordinary ray is no longer orthogonal to its wave vector. However, the polarizations of the electric field stated in Eq. (34) are orthogonal to the wave vector, which is why an extraordinary ray cannot be identified in this setting. The two polarizations only split due to their distinct propagation velocities, cf. Eq. (30). Therefore, the polarization plane of a linearly polarized wave will rotate indicating an optically active material. This phenomena is quantified by defining the specific rotatory power $\Delta$, measuring the rotation of the plane of linearly polarized light per unit traversed length in

\footnotetext{
${ }^{3}$ We define a polarization as right-handed (left-handed) if the polarization vector of a plane wave rotates along a circle in the clockwise (counterclockwise) direction when the observer is facing into the incoming wave $[30,33]$.
}

the medium. It is given by $\Delta \equiv-(\Delta n) \omega / 2$ where $\Delta n \equiv$ $n_{+}-n_{-}$is the difference of refractive indices for the two polarization directions. In our case, the latter definition yields

$$
\Delta=-\frac{\mu \Sigma}{2},
$$

thus providing a frequency-independent specific rotatory power due to the chiral magnetic conductivity $\Sigma$.

As we know, there is no clear connection between the $\mathrm{CME}$ and measurements of birefringence, and so we discuss a possible way to observe this effect. Before more elaborate methods were developed, birefringence was detected with a set of crossed polarizers that does not permit any light to pass. If a birefringent material is put in between these two polarizers, it rotates the polarization plane of the light that passed the first polarizer such that light of a nonvanishing intensity can be measured behind the second polarizer. However, this method is not precise enough to measure small values of birefringence. Furthermore, it is challenging to measure birefringence of an inhomogeneous medium.

Therefore, a more sophisticated technique was developed in Ref. [34]. The system to be used is based on a rotating polarizer, a quarter-wave plate, and an analyzer. The passing light is captured by a CCD camera whereupon the measured data are evaluated by a computer. This system was commercially distributed under the name Metripol.

The intensity $I$ of the light detected at any point is expressed in terms of angles $\xi$ and $\psi$ describing the orientation of the rotating polarizer:

$$
I=\frac{I_{0}}{2}[1-\sin (2 \xi-2 \psi) \sin \delta],
$$

where $I_{0} / 2$ is a suitably normalized intensity and $\delta$ is the phase shift between the two physical polarizations of the light. The latter is

$$
\delta=\frac{2 \pi}{\lambda} d(\Delta n),
$$

where $d$ is the sample thickness and $\lambda$ is the vacuum wavelength of the incoming light.

Thus, to measure birefringence of a material endowed with a magnetic conductivity, a sample of thickness $d$ can be placed into a Metripol system to determine the factor $\sin \delta$. This factor provides the difference between the refractive indices via $\Delta n=\delta \lambda /(2 \pi d)$. The latter experimental result in combination with Eq. (25) leads to the magnetic-conductivity parameter:

$$
\Sigma=\frac{\omega(\Delta n)}{\mu} .
$$

A similar technique for birefringence measurements was also reported in Ref. [35]. 


\section{B. Diagonal anisotropic chiral conductivity}

As a next step, we will consider another particular case for the chiral conductivity, $\sigma_{i j}^{B}$, which is represented by a diagonal tensor that describes an anisotropic system [15]:

$$
\left[\sigma_{i j}^{B}\right]=\left(\begin{array}{ccc}
\Sigma_{x} & 0 & 0 \\
0 & \Sigma_{y} & 0 \\
0 & 0 & \Sigma_{z}
\end{array}\right),
$$

with a set $\{\Sigma\}=\left\{\Sigma_{x}, \Sigma_{y}, \Sigma_{z}\right\}$ of distinct elements in the diagonal, $\Sigma_{x} \neq \Sigma_{y} \neq \Sigma_{z}$. The general permittivity tensor is given by Eq. (12b), with the components shown as follows:

$$
\left[\bar{\epsilon}_{i j}\right]=\left(\begin{array}{ccc}
\epsilon+\mathrm{i} \frac{\sigma}{\omega} & -\frac{\mathrm{i}}{\omega^{2}} \Sigma_{x} k_{3} & \frac{\mathrm{i}}{\omega^{2}} \Sigma_{x} k_{2} \\
\frac{\mathrm{i}}{\omega^{2}} \Sigma_{y} k_{3} & \epsilon+\mathrm{i} \frac{\sigma}{\omega} & -\frac{\mathrm{i}}{\omega^{2}} \Sigma_{y} k_{1} \\
-\frac{\mathrm{i}}{\omega^{2}} \Sigma_{z} k_{2} & \frac{\mathrm{i}}{\omega^{2}} \Sigma_{z} k_{1} & \epsilon+\mathrm{i} \frac{\sigma}{\omega}
\end{array}\right) .
$$

The dispersion relations are obtained from $\operatorname{det}\left[M_{i j}\right]=0$ where the tensor $M_{i j}$ is defined in Eq. (15b), with $\bar{\epsilon}_{i j}$ given by Eq. (40). Evaluating this condition leads to the dispersion equation

$$
\left[\omega n^{2}-\mu(\mathrm{i} \sigma+\epsilon \omega)\right]^{2}=\Omega
$$

with the function

$$
\Omega=\Omega(\mathbf{n})=\mu^{2}\left(\Sigma_{x} \Sigma_{y} n_{3}^{2}+\Sigma_{x} \Sigma_{z} n_{2}^{2}+\Sigma_{y} \Sigma_{z} n_{1}^{2}\right) .
$$

The latter is an involved expression, since it explicitly contains the components of the vector $\mathbf{n}=\left(n_{1}, n_{2}, n_{3}\right)$ instead of its modulus $n$ as in the isotropic case investigated previously. To avoid problems with separating real and imaginary parts of refractive indices, we will assume that $\Omega \geq 0$. The following parametrization [30] for $\mathbf{n}$ permits a convenient examination of the physical content of Eq. (41a):

$$
\mathbf{n}=n(\sin \theta \cos \phi, \sin \theta \sin \phi, \cos \theta) \equiv n \mathbf{m},
$$

with angles $\theta \in[0, \pi]$ and $\phi \in[0,2 \pi)$. With the latter, Eq. (41a) is rewritten as

$$
\omega^{2} n^{4}-D n^{2}-G=0,
$$

where

$$
\begin{gathered}
D=2 \mu \omega(\epsilon \omega+\mathrm{i} \sigma)+\tilde{\Omega}, \\
G=\mu^{2}\left(\sigma^{2}-2 \mathrm{i} \epsilon \omega \sigma-\epsilon^{2} \omega^{2}\right),
\end{gathered}
$$

$$
\begin{aligned}
\tilde{\Omega}= & \mu^{2}\left[\cos ^{2} \theta \Sigma_{x} \Sigma_{y}+\sin ^{2} \theta \sin ^{2} \phi \Sigma_{x} \Sigma_{z}\right. \\
& \left.+\sin ^{2} \theta \cos ^{2} \phi \Sigma_{y} \Sigma_{z}\right]=\Omega / n^{2} .
\end{aligned}
$$

The dispersion equation (43) yields two solutions for $n$,

$$
n_{ \pm}^{2}=\frac{D}{2 \omega^{2}} \pm \frac{1}{2 \omega^{2}} \sqrt{D^{2}+4 \omega^{2} G},
$$

revealing two values for any frequency.

\section{Dielectric nonconducting medium}

In the special limit of zero Ohmic conductivity, $\sigma=0$, all the complex pieces of Eq. (41a) or Eq. (43) vanish and the refractive indices of Eq. (44) are real. Some complex term could occur in Eq. (44) if the discriminant is negative: $\Delta_{0}=D_{0}^{2}+4 \omega^{2} G_{0}<0$, where $G_{0}=-\epsilon^{2} \mu^{2} \omega^{2}$ and $D_{0}=2 \epsilon \mu \omega^{2}+\tilde{\Omega}$. But $\Delta_{0}=\tilde{\Omega}^{2}+4 \tilde{\Omega} \epsilon \mu \omega^{2}>0$ and therefore, this possibility is not realized. In fact, for $\sigma=0$, Eq. (41a) gives rise to

$$
\omega^{2}\left(n^{2}-\mu \epsilon\right)^{2}=\Omega
$$

whereupon

$$
n_{ \pm}^{2}=\mu \epsilon \pm \frac{1}{\omega} \sqrt{\Omega} .
$$

If we apply the prescription stated in Eq. (42) to Eq. (44), the refractive indices are given by

$$
n_{ \pm}^{2}=\mu \epsilon+\frac{\tilde{\Omega}}{2 \omega^{2}} \pm \frac{1}{2 \omega^{2}} \sqrt{4 \mu \epsilon \omega^{2} \tilde{\Omega}+\tilde{\Omega}^{2}} .
$$

It is interesting to observe that by using the parametrization of Eq. (42) in each refractive index of Eq. (46) one obtains an explicit solution for $n_{ \pm}$. Each equation is of second order and the refractive indices are

$$
\begin{gathered}
n_{ \pm}=\sqrt{2 \Upsilon_{\{\Sigma\}}} \pm \frac{\sqrt{\tilde{\Omega}}}{2 \omega}, \\
2 \Upsilon_{\{\Sigma\}}=\mu \epsilon+\frac{\tilde{\Omega}}{4 \omega^{2}},
\end{gathered}
$$

where one easily verifies that their squares reproduce the results in Eq. (47). Hence, for $\sigma=0, \sigma_{i j}^{B} \neq 0$, we have a dielectric nonconducting and dispersive medium. One can verify that Eq. (45) provides three different expressions for the following three situations: $\mathbf{n}=\left(0, n_{2}, n_{3}\right), \quad \mathbf{n}=$ $\left(n_{1}, 0, n_{3}\right)$, and $\mathbf{n}=\left(n_{1}, n_{2}, 0\right)$. For each of these three choices, Eq. (46) yields two values for $n$, resulting in birefringence.

Diagonalizing the EPT of Eq. (40) for zero Ohmic conductivity $(\sigma=0)$ and dropping the parametrization given by Eq. (42), one obtains $\epsilon_{1}=\epsilon$ and 


$$
\epsilon_{2,3}=\epsilon \pm \frac{\gamma}{\omega^{2}}
$$

with

$$
\begin{aligned}
\gamma(\mathbf{k}) & \equiv \frac{\omega}{\mu} \sqrt{\Omega(\mathbf{k} / \omega)} \\
& =\sqrt{\Sigma_{y} \Sigma_{z} k_{1}^{2}+\Sigma_{x} \Sigma_{z} k_{2}^{2}+\Sigma_{x} \Sigma_{y} k_{3}^{2}} .
\end{aligned}
$$

In this case, when the two eigenvalues of the EPT, given in Eq. (49a), are multiplied by $\mu$, we obtain $\mu \epsilon_{2,3}=\mu \epsilon_{ \pm}$, being equal to the values for $n_{ \pm}^{2}$ in Eq. (46) calculated from the condition $\operatorname{det}\left[M_{i j}\right]=0$. This numerical coincidence provides a nice example of the fact that even though we satisfy the condition $n_{ \pm}^{2}=\mu \epsilon_{ \pm}$, this equality does not imply that the associated eigenvectors of the EPT would necessarily correspond to the propagation modes of the electric field. The corresponding eigenvectors are

$$
\begin{aligned}
& \mathbf{e}_{1}=\left(\begin{array}{c}
k_{1} / k_{3} \\
k_{2} / k_{3} \\
1
\end{array}\right), \\
& \mathbf{e}_{2}= \frac{1}{\varkappa}\left(\begin{array}{c}
\mathrm{i} \Sigma_{x} k_{2} \gamma-\Sigma_{x} \Sigma_{y} k_{1} k_{3} \\
-\mathrm{i} \Sigma_{y} k_{1} \gamma-\Sigma_{x} \Sigma_{y} k_{2} k_{3} \\
\varkappa
\end{array}\right), \\
& \mathbf{e}_{3}=\frac{1}{\varkappa}\left(\begin{array}{c}
-\mathrm{i} \Sigma_{x} k_{2} \gamma-\Sigma_{x} \Sigma_{y} k_{1} k_{3} \\
\mathrm{i} \Sigma_{y} k_{1} \gamma-\Sigma_{x} \Sigma_{y} k_{2} k_{3} \\
\varkappa
\end{array}\right),
\end{aligned}
$$

where

$$
\begin{aligned}
\varkappa & =\Sigma_{z}\left(\Sigma_{y} k_{1}^{2}+\Sigma_{x} k_{2}^{2}\right) \\
& =\omega^{2} n^{2} \Sigma_{z}\left(\Sigma_{y} m_{1}^{2}+\Sigma_{x} m_{2}^{2}\right),
\end{aligned}
$$

with $\mathbf{m}$ defined in Eq. (42). Note that

$$
\begin{aligned}
\mathbf{e}_{1} \cdot \mathbf{e}_{2}^{*}= & 1-\frac{\left(k_{1}^{2}+k_{2}^{2}\right) \Sigma_{x} \Sigma_{y}}{\varkappa} \\
& -\mathrm{i} \frac{\gamma k_{1} k_{2}\left(\Sigma_{x}-\Sigma_{y}\right)}{\varkappa k_{3}}, \\
\mathbf{e}_{1} \cdot \mathbf{e}_{3}^{*}= & \mathbf{e}_{1}^{*} \cdot \mathbf{e}_{2}, \\
\mathbf{e}_{2} \cdot \mathbf{e}_{3}^{*}= & 1-\frac{\Sigma_{x}^{2}\left(\gamma k_{2}+\mathrm{i} k_{1} k_{3} \Sigma_{y}\right)^{2}}{\varkappa^{2}} \\
& +\frac{\Sigma_{y}^{2}\left(k_{2} k_{3} \Sigma_{x}+\mathrm{i} \gamma k_{1}\right)^{2}}{\varkappa^{2}} .
\end{aligned}
$$

The above calculation shows that $\mathbf{k} \cdot \mathbf{e}_{a} \neq 0$ for all $a=1$, 2,3 in such a way that these eigenvectors cannot describe the propagation modes $\mathbf{E}_{ \pm}$of the electric field. In order to clearly illustrate this point, we calculate such propagation modes for the particular case where $\mathbf{k}=\left(k_{1}, k_{2}, 0\right)=$ $\omega n\left(m_{1}, m_{2}, 0\right)$ and compare those with the corresponding eigenvectors of the EPT. The results for the propagation modes are

$$
\mathbf{E}_{ \pm}=\left(\begin{array}{c}
-\omega n_{ \pm} m_{2} / u_{ \pm} \\
\omega n_{ \pm} m_{1} / v_{ \pm} \\
\pm \mathrm{i} \omega^{2} \Sigma_{z} / \sqrt{\varkappa_{ \pm}}
\end{array}\right)
$$

with

$$
\begin{aligned}
& u_{ \pm}=\frac{\mu \epsilon}{1 \mp \mu \Sigma_{x} \Sigma_{z} / \sqrt{\varkappa_{ \pm}}}, \\
& v_{ \pm}=\frac{\mu \epsilon}{1 \mp \mu \Sigma_{y} \Sigma_{z} / \sqrt{\varkappa_{ \pm}}}, \\
& \varkappa_{ \pm}=\omega^{2} n_{ \pm}^{2} \Sigma_{z}\left(\Sigma_{y} m_{1}^{2}+\Sigma_{x} m_{2}^{2}\right) .
\end{aligned}
$$

The refractive indices satisfy the quadratic equation

$$
n_{ \pm}^{2}=\mu\left(\epsilon \pm \frac{n_{ \pm}}{\omega} \sqrt{\Sigma_{z}\left(\Sigma_{x} m_{2}^{2}+\Sigma_{y} m_{1}^{2}\right)}\right)
$$

which is written in terms of the unit vector $\mathbf{m}$ defining the planes of constant phase of the wave. The dispersion relations $\omega_{ \pm}=\omega_{ \pm}(\mathbf{k})$ are

$$
\omega_{ \pm}=\frac{1}{\sqrt{\mu \epsilon}} \sqrt{k_{1}^{2}+k_{2}^{2} \mp \mu \sqrt{\Sigma_{z}\left(\Sigma_{x} k_{2}^{2}+\Sigma_{y} k_{1}^{2}\right)}} .
$$

The functions $u_{ \pm}$and $v_{ \pm}$satisfy the useful relations

$$
n_{ \pm}^{2}\left(\frac{m_{1}^{2}}{v_{ \pm}}+\frac{m_{2}^{2}}{u_{ \pm}}\right)=1
$$

which are just a consequence of Eq. (54). We observe that

$$
\mathbf{m} \cdot \mathbf{E}_{ \pm}= \pm \epsilon^{-1} m_{1} m_{2} \frac{\left(\Sigma_{x}-\Sigma_{y}\right) \sqrt{\Sigma_{z}}}{\sqrt{\Sigma_{y} m_{1}^{2}+\Sigma_{x} m_{2}^{2}}}
$$

Due to $\left.\gamma\right|_{k_{3}=0}=\sqrt{\varkappa}$, from Eqs. (50b) and (50c) we read that in this case the eigenvectors of the EPT are

$$
\begin{aligned}
& \mathbf{e}_{ \pm} \equiv \pm \mathrm{i} \sqrt{\varkappa} \mathbf{e}_{2,3}=\left(\begin{array}{c}
-\Sigma_{x} k_{2} \\
\Sigma_{y} k_{1} \\
\pm \mathrm{i} \sqrt{\varkappa}
\end{array}\right), \\
& \epsilon_{ \pm}=\epsilon \pm \frac{\sqrt{\varkappa}}{\omega^{2}}
\end{aligned}
$$


with $\varkappa$ of Eq. (51). The latter $\mathbf{e}_{ \pm}$do not coincide with $\mathbf{E}_{ \pm}$of Eq. (53). The propagating modes of Eq. (53) are not orthogonal to the wave vector $\mathbf{k}$. This is a characteristic of wave propagation in anisotropic media, which nevertheless must always fulfill $\mathbf{k} \cdot \mathbf{D}=0$ in the absence of sources.

To have a direct physical interpretation of the propagating modes in Eq. (53), we perform another simplification and consider the case of propagation along the $y$ axis, i.e., we take $\mathbf{m}=(0,1,0)$. Now we have $n_{ \pm}^{2}=u_{ \pm}$and $\sqrt{\varkappa_{ \pm}}=\omega n_{ \pm} \sqrt{\Sigma_{x} \Sigma_{z}}$. After substituting in Eq. (53), we obtain the normalized electric fields

$$
\left.\hat{\mathbf{E}}_{ \pm}\right|_{m_{1}=m_{3}=0}=\sqrt{\frac{\Sigma_{x}}{\Sigma_{x}+\Sigma_{z}}}\left(\begin{array}{c}
1 \\
0 \\
\mp \mathrm{i} \sqrt{\Sigma_{z} / \Sigma_{x}}
\end{array}\right),
$$

which describe elliptically polarized modes with distinct refractive indices

$$
n_{ \pm}=\sqrt{\mu \epsilon+\frac{\mu^{2}}{4 \omega^{2}} \Sigma_{x} \Sigma_{z}} \pm \frac{\mu}{2 \omega} \sqrt{\Sigma_{x} \Sigma_{z}} .
$$

The latter correspond to specific cases of Eq. (48). The modes associated with $\hat{\mathbf{E}}_{ \pm}$in Eq. (59) represent the lefthanded and right-handed polarization states via the same definition used at the end of Sec. III A. It is relevant to point out that the propagation of the wave associated with Eq. (59) occurs along the $y$ axis, while the wave connected to Eq. (34) propagates along the $z$ axis. This explains the opposite signs that appear in the polarization modes of Eq. (59) in comparison with those of Eq. (34).

Making cyclic changes among $\{x, y, z\}$, we identify $n_{ \pm}=n_{L, R}$, thus characterizing an optically active medium. Note that in this particular case we have $\mathbf{k} \cdot \hat{\mathbf{E}}_{ \pm}=0$, so we expect $\hat{\mathbf{E}}_{ \pm}$to be proportional to the eigenvectors $\mathbf{e}_{ \pm}$in Eq. (58a), as can be readily verified.

It is also easy to notice that Eq. (48) together with Eq. (60) include the isotropic case. Indeed, assuming that $\Sigma_{x}=\Sigma_{y}=\Sigma_{z}=\Sigma$, we have $\tilde{\Omega}=\mu^{2} \Sigma^{2}$ and Eq. (48) exactly reproduces Eq. (25). Also, we recover the circularly polarized modes of Eq. (34), though with respect to a different axis. Both the isotropic and anisotropic nonconducting diagonal cases exhibit birefringence.

Still, a main difference arises: in the former case, the two refractive indices are independent of the direction defined by the wave vector $\mathbf{k}$ [see Eq. (24)], while in the latter case a direction dependence emerges due to the appearance of $\tilde{\Omega}$ in Eq. (47). Nevertheless, $\tilde{\Omega}$ is a quadratic function of the components of the wave vector $\mathbf{k}$, which yields a parityinvariant individual refractive index. As in the isotropic case, parity violation manifests itself in the different refractive indices of the left- and right-handed modes. Another remarkable feature of the diagonal anisotropic case, which will also appear in the instances discussed below, is the presence of a non-Hermitian EPT. Although the latter opens up interesting new possibilities, we defer related analyses to future work.

Finally, it is intriguing to notice that a diagonal chiral conductivity tensor inserted into the Maxwell equations is not enough to establish a conducting behavior for a dielectric medium although this is the chiral conductivity configuration most used and cited in the literature.

\section{Nondiagonal antisymmetric conductivity}

Now we analyze the case where the magnetic conductivity is described by an exotic configuration given by an antisymmetric tensor, $\sigma_{i j}^{B}$, parametrized in terms of a threevector, $\mathbf{b}=\left(b_{1}, b_{2}, b_{3}\right)$, as

$$
\sigma_{i j}^{B}=\epsilon_{i j k} b_{k},
$$

with the Levi-Civita symbol $\epsilon_{i j k}$ in three dimensions. Notice that this case corresponds to the situation in magnetohydrodynamics of an isotropic resistivity in Eq. (1) and the vector $\mathbf{b}$ is proportional to the plasma velocity. Inserting Eq. (61) into Eq. (12b), leads to the EPT

$$
\bar{\epsilon}_{i j}(\omega)=\left(\epsilon+\mathrm{i} \frac{\sigma}{\omega}+\mathrm{i} \frac{\mathbf{k} \cdot \mathbf{b}}{\omega^{2}}\right) \delta_{i j}-\frac{\mathrm{i}}{\omega^{2}} k_{i} b_{j} .
$$

By employing Eq. (62) in Eq. (15b), one obtains

$$
\begin{aligned}
{\left[M_{i j}\right]=} & \left(\begin{array}{ccc}
n_{2}^{2}+n_{3}^{2}-\mu \epsilon & -n_{1} n_{2} & -n_{1} n_{3} \\
-n_{1} n_{2} & n_{1}^{2}+n_{3}^{2}-\mu \epsilon & -n_{2} n_{3} \\
-n_{1} n_{3} & -n_{2} n_{3} & n_{1}^{2}+n_{2}^{2}-\mu \epsilon
\end{array}\right) \\
& +\mathrm{i} \frac{\mu}{\omega}\left(\begin{array}{ccc}
-\left(\sigma+n_{2} b_{2}+n_{3} b_{3}\right) & n_{1} b_{2} & n_{1} b_{3} \\
n_{2} b_{1} & -\left(\sigma+n_{1} b_{1}+n_{3} b_{3}\right) & n_{2} b_{3} \\
n_{3} b_{1} & n_{3} b_{2} & -\left(\sigma+n_{1} b_{1}+n_{2} b_{2}\right)
\end{array}\right),
\end{aligned}
$$


for which $\operatorname{det}\left[M_{i j}\right]=0$ implies

$$
\left[n^{2}-\mathrm{i} \frac{\mu}{\omega}(\mathbf{b} \cdot \mathbf{n})-\mu\left(\epsilon+\mathrm{i} \frac{\sigma}{\omega}\right)\right]^{2}=0 .
$$

Contrary to Eqs. (43) and (45), the latter dispersion equation involves the square of a quadratic polynomial in the components of $\mathbf{n}$. Hence, the solution for the refractive index is doubly degenerate and there is only a single refractive index with a non-negative real part. Implementing $\mathbf{b} \cdot \mathbf{n}=b n \cos \theta$ with $b=|\mathbf{b}|$, Eq. (64) provides the following refractive index:

$$
\begin{gathered}
n=\sqrt{2 \Upsilon_{b}+\mathrm{i} \mu \frac{\sigma}{\omega}}+\mathrm{i} \Xi_{b}, \\
2 \Upsilon_{b}=\mu \epsilon-\Xi_{b}^{2}, \quad \Xi_{b}=\frac{\mu}{2 \omega} b \cos \theta .
\end{gathered}
$$

Note the presence of the minus sign between the two contributions in $\Upsilon_{b}$ in contrast to $\Upsilon_{\Sigma}$ of Eq. (22b) and $\Upsilon_{\{\Sigma\}}$ in Eq. (48b). Thus, we assume that $\mu \epsilon \geq \Xi_{b}^{2}$. Decomposing the latter refractive index into its real and imaginary parts implies

$$
n=\alpha_{+}^{\prime}+\mathrm{i}\left(\Xi_{b}+\alpha_{-}^{\prime}\right)
$$

where

$$
\alpha_{ \pm}^{\prime}=\sqrt{\sqrt{\Upsilon_{b}^{2}+\left(\frac{\mu \sigma}{2 \omega}\right)^{2}} \pm \Upsilon_{b}}
$$

In this case, we obtain an anisotropic complex refractive index that captures the effects of the exotic conductivity, as shown in Eq. (66a), which is compatible with a conducting medium. The imaginary part takes the role of the absorption coefficient for the electromagnetic wave, which undergoes attenuation while it propagates. Therefore, an electromagnetic wave cannot propagate through such a medium, since absorption damps its intensity. When considered in a dielectric conducting medium, $\left(\epsilon, \sigma, \sigma^{B}\right)$, the magnetic conductivity modifies the real and imaginary parts of the refractive index. This causes a modification of the absorption coefficient given by $\tilde{\alpha}=\omega \alpha_{-}^{\prime}+\mu b \cos \theta$, where $\alpha_{-}^{\prime}$ is stated in Eq. (66b).

Note that for a diagonal magnetic conductivity there were two distinct refractive indices with a positive real part; see Eqs. (25) and (48). Thus, the occurrence of a single refractive index in Eq. (65) is quite unexpected in the context of a parity-violating theory. In contrast to the cases studied before, a magnetic conductivity given by Eq. (61) does not imply birefringence. These results suggest that an antisymmetric magnetic conductivity leads to an EPT $\bar{\epsilon}_{i j}$ of a form that permits only a single refractive index with a positive real part. In the following section, we will calculate the propagation modes to get a definite prediction.

A parity transformation in three spatial dimensions implies $\cos \theta \mapsto \cos (\pi-\theta)=-\cos \theta$ for the polar angle (and $\phi \mapsto \pi+\phi$ for the azimuthal angle, which does not occur in the refractive index). As the real part of the refractive index in Eq. (65) only contains squares of $\cos \theta$, it is invariant under parity transformations. However, at least the imaginary part of $n$ exhibits parity-violating properties.

We note in passing that there is a direct connection between the configuration under consideration and the material studied in Ref. [36]. We call attention to Eq. (2) in the latter paper that describes a magnetic conductivity for the material TaAs. This means that the authors of Ref. [36] have found a microscopic realization of a crystal that leads to a macroscopic, effective magnetic conductivity of the form of our Eq. (61) (with the identification $\sigma_{B} \hat{c}_{i}=-b_{i}$ with their $\sigma_{B}$ and $\hat{c}$ ).

Our understanding of their result is as follows. They employed a sample-based coordinate system $(a, b, c)$ and the crystal lattice of TaAs has symmetries with respect to certain axes and planes of this coordinate system. There is a second-rank tensor $\sigma_{P}$ that links components of the current $\mathbf{J}$ and angular momentum $\mathbf{L}: J^{i}=\left(\sigma_{P}\right)^{i}{ }_{k}{ }^{k}$. The nonzero components of $\sigma_{P}$ are determined from the symmetry properties of the crystal. The current $\mathbf{J}$ transforms like a vector under parity transformations and reflections at single planes, whereas the angular momentum $\mathbf{L}$ transforms like a pseudovector.

Let us consider the $b-c$ plane. The above relation links components of the current and angular momentum in this plane: $J^{b}=\left(\sigma_{P}\right)^{b}{ }_{c} L^{c}$. If there is a reflection symmetry with respect to the $b-c$ plane, the vectors transform according to the set of (un-numbered) transformation rules given under Eq. (1) in Ref. [36]. If $J^{b}$ is then linked to $L^{c}$ by a nonzero $\left(\sigma_{P}\right)^{b}{ }_{c}$, there will be a contradiction, as the components of $\mathbf{J}$ in the plane transform differently from the components of $\mathbf{L}$ in the same plane. So the corresponding component of the tensor $\sigma_{P}$ must be zero. These arguments imply a magnetic conductivity of TaAs in the form of Eq. (61).

\section{Dielectric nonconducting medium}

Let us come back to Eq. (65). If we start from a dielectric with zero Ohmic conductivity $\left(\epsilon \neq 0, \sigma=0, \sigma^{B} \neq 0\right)$, the latter equations reduce to

$$
n=\sqrt{2 \Upsilon_{b}}+\mathrm{i} \Xi_{b}, \quad \Xi_{b}=\frac{\mu b_{3}}{2 \omega},
$$

which is compatible with the behavior of a conducting medium. Therefore, the off-diagonal chiral conductivity of

\footnotetext{
${ }^{4}$ Note that we employ symbols different from those in Ref. [36] to avoid confusion with some of our quantities.
} 
Eq. (61) ascribes a conducting behavior to the material even for a purely dielectric substrate $(\epsilon \neq 0, \sigma=0)$.

\section{Propagation modes}

For a phenomenological analysis of the physics, let us choose a convenient coordinate system, without loss of generality. Since $\mathbf{n}$ and $\mathbf{b}$ define a plane, to be labeled the $y-z$ plane, we take the $z$ axis along the direction of $\mathbf{n}$ so that

$$
\mathbf{n}=(0,0, n), \quad \mathbf{b}=b(0, \sin \theta, \cos \theta) \equiv\left(0, b_{2}, b_{3}\right) .
$$

Recall that $\theta$ is the angle between $\mathbf{n}$ and $\mathbf{b}$. This choice of coordinates leads to a very simple expression for the matrix of Eq. (63):

$$
\left[M_{i j}\right]=\left(\begin{array}{ccc}
n^{2}-\mu \epsilon-n \frac{\mathrm{i} \mu}{\omega} b_{3} & 0 & 0 \\
0 & n^{2}-\mu \epsilon-n \frac{\mathrm{i} \mu}{\omega} b_{3} & 0 \\
0 & n \frac{\mathrm{i} \mu}{\omega} b_{2} & -\mu \epsilon
\end{array}\right),
$$

which immediately yields the dispersion equation

$$
\left(n^{2}-\mu \epsilon-n \frac{\mathrm{i} \mu}{\omega} b_{3}\right)^{2}=0,
$$

with $n b_{3}=\mathbf{n} \cdot \mathbf{b}$. The latter corresponds exactly to Eq. (64) when $\sigma=0$. Equation (70) has a single solution with a nonnegative real part, given by Eq. (67).

Recalling that $\sqrt{2 \Upsilon_{b}}=\sqrt{\mu \epsilon-\Xi_{b}^{2}}$, we distinguish between two cases according to the choice of the sign inside the square root of the above relation. In the first case, when $\mu \epsilon \leq \Xi_{b}^{2}$, the refractive index is purely imaginary with a positive imaginary part, which damps propagation. The alternative, $\mu \epsilon>\Xi_{b}^{2}$, yields propagation modes that are to be described now. The condition $M_{i j} E^{j}=0$ requires

$$
E^{3}=n \frac{\mathrm{i} b_{2}}{\epsilon \omega} E^{2},
$$

leaving $E^{1}$ completely arbitrary. We take advantage of this freedom to choose two orthogonal vectors satisfying the condition in Eq. (71). We find

$$
\begin{aligned}
\mathbf{E}_{ \pm} & =\frac{1}{\sqrt{2\left(1+Q^{2}\right)}}\left(\begin{array}{c} 
\pm \sqrt{1+Q^{2}} \\
-1 \\
-\mathrm{i} Q e^{\mathrm{i} \alpha}
\end{array}\right) \\
Q & =\frac{b_{2} N}{\epsilon \omega} .
\end{aligned}
$$

Here we parametrized the complex refractive index as

$$
\begin{gathered}
n=N e^{\mathrm{i} \alpha}, \quad N=\sqrt{n^{*} n}=\sqrt{\mu \epsilon}, \\
\tan \alpha=\frac{\Xi_{b}}{\sqrt{\mu \epsilon-\left(\Xi_{b}\right)^{2}}} .
\end{gathered}
$$

We can easily verify that $\mathbf{E}_{+}^{*} \cdot \mathbf{E}_{-}=0$ and also that the $y$ and $z$ components of the propagation modes obey Eq. (71). Thus, we have recovered two orthogonal modes whose propagation is described by the same refractive index. This is an unexpected result, which nevertheless is analogous to the simplest isotropic case (without the presence of a magnetic conductivity) where two linear polarization modes associated with the same refractive index occur. Notice that we have $\mathbf{k} \cdot \mathbf{E}_{ \pm} \neq 0$ in this case, which prevents an interpretation of the fields in terms of standard elliptical polarizations with components defined in the twodimensional subspace orthogonal to $\mathbf{k}$.

Since in the coordinate system defined in Eq. (68) we only have access to the inversion $z \mapsto-z$, the parity transformation $\mathbf{n} \mapsto-\mathbf{n}$ can be better studied in a rotated frame where now the vector $\mathbf{b}$ defines the new $z$ axis endowing the system with axial symmetry. The new frame is obtained via a rotation of the former one by an angle $\theta=\arccos \left(b_{3} /|\mathbf{b}|\right)$ with respect to an axis perpendicular to the n-b plane. The latter can be associated with an arbitrary plane having a constant azimuthal angle $\phi$ in spherical coordinates. Calling $\bar{E}^{i}$ the components of the electric field in the rotated frame, we derive the following expressions:

$\bar{E}_{ \pm}^{1}= \pm \frac{1}{\sqrt{2}}$

$\bar{E}_{ \pm}^{2}=-\frac{1}{\sqrt{2\left(1+\bar{Q}^{2} \sin ^{2} \theta\right)}}\left[\cos \theta-\mathrm{i} \bar{Q} e^{\mathrm{i} \alpha} \sin ^{2} \theta\right]$,

$\bar{E}_{ \pm}^{3}=-\frac{1}{\sqrt{2\left(1+\bar{Q}^{2} \sin ^{2} \theta\right)}} \sin \theta\left[1+\mathrm{i} \bar{Q} e^{\mathrm{i} \alpha} \cos \theta\right]$,

with

$$
\bar{Q}=\frac{b N}{\epsilon \omega} .
$$

The magnetic fields corresponding to Eq. (72) are obtained using $\mathbf{B}_{ \pm}=\mathbf{n} \times \mathbf{E}_{ \pm}$and those associated with Eq. (74) are computed in an analog way.

As $\mathbf{n}$ lies in a plane, we restrict the parity transformation to the replacement rule of the polar angle: $\theta \mapsto \pi-\theta$. Then, a parity transformation of a vector in two dimensions corresponds to its reflection at one of the two axes modulo a rotation by $\pi$. Thus, the expected behavior under a parity transformation in the plane is that only one of the two vector components flips its sign. The vector $\mathbf{n}$ is transformed in a way that the $n_{3}$ component is reflected at the $n_{2}$ axis. If parity was conserved, the sign of the second components $\bar{E}_{ \pm}^{2}$ should change under a parity 
transformation, while the third components $\bar{E}_{+}^{3}$ should remain invariant. However, one can verify that $\bar{E}_{ \pm}^{2}$ and $\bar{E}_{ \pm}^{3}$ do not behave in this manner. On the contrary, the terms involving $b$ spoil the behavior expected, which is a signal of parity violation. Note that the refractive indices of Eqs. (65a) and (67) also change under parity transformations, as $\Xi_{b} \mapsto-\Xi_{b}$.

\section{Nondiagonal symmetric conductivity tensor}

Now we examine the case where the magnetic conductivity is given by a traceless symmetric tensor, in accordance with the following parametrization:

$$
\sigma_{i j}^{B}=\frac{1}{2}\left(a_{i} c_{j}+a_{j} c_{i}\right)
$$

where $a_{i}$ and $c_{i}$ are the components of two orthogonal background vectors $\mathbf{a}$ and $\mathbf{c}$, i.e., $\mathbf{a} \cdot \mathbf{c}=0$ such that $\sigma_{i i}^{B}=0$. Inserting Eq. (75) into Eq. (12b) yields

$$
\bar{\epsilon}_{i j}=\left(\epsilon+\mathrm{i} \frac{\sigma}{\omega}\right) \delta_{i j}+\frac{\mathrm{i}}{2 \omega^{2}}\left(a_{i} c_{n}+a_{n} c_{i}\right) \epsilon_{n b j} k_{b} .
$$

The tensor stated in Eq. (15b) is explicitly represented by the following matrix:

$$
\begin{aligned}
{\left[M_{i j}\right]=} & \left(\begin{array}{ccc}
n_{2}^{2}+n_{3}^{2}-\mu \epsilon & -n_{1} n_{2} & -n_{1} n_{3} \\
-n_{1} n_{2} & n_{1}^{2}+n_{3}^{2}-\mu \epsilon & -n_{2} n_{3} \\
-n_{1} n_{3} & -n_{2} n_{3} & n_{1}^{2}+n_{2}^{2}-\mu \epsilon
\end{array}\right) \\
& -\mathrm{i} \frac{\mu}{2 \omega}\left(\begin{array}{ccc}
2 \sigma+\epsilon_{11} & n_{1}\left(a_{1} c_{3}+a_{3} c_{1}\right)-2 n_{3} a_{1} c_{1} & -n_{1}\left(a_{1} c_{2}+a_{2} c_{1}\right)+2 n_{2} a_{1} c_{1} \\
-n_{2}\left(a_{2} c_{3}+a_{3} c_{2}\right)+2 n_{3} a_{2} c_{2} & 2 \sigma+\epsilon_{22} & n_{2}\left(a_{2} c_{1}+a_{1} c_{2}\right)-2 n_{1} a_{2} c_{2} \\
n_{3}\left(a_{3} c_{2}+a_{2} c_{3}\right)-2 n_{2} a_{3} c_{3} & -n_{3}\left(a_{3} c_{1}+a_{1} c_{3}\right)+2 n_{1} a_{3} c_{3} & 2 \sigma+\epsilon_{33}
\end{array}\right),
\end{aligned}
$$

where

$$
\begin{aligned}
& \epsilon_{11}=\left(a_{1} c_{2}+a_{2} c_{1}\right) n_{3}-\left(a_{1} c_{3}+a_{3} c_{1}\right) n_{2}, \\
& \epsilon_{22}=\left(a_{2} c_{3}+a_{3} c_{2}\right) n_{1}-\left(a_{1} c_{2}+a_{2} c_{1}\right) n_{3}, \\
& \epsilon_{33}=\left(a_{3} c_{1}+a_{1} c_{3}\right) n_{2}-\left(a_{3} c_{2}+a_{2} c_{3}\right) n_{1} .
\end{aligned}
$$

The evaluation of $\operatorname{det}\left[M_{i j}\right]=0$ yields the dispersion equation stated as follows:

$$
\begin{aligned}
0= & {\left[n^{2}-\mu\left(\epsilon+\mathrm{i} \frac{\sigma}{\omega}\right)+\mathrm{i} \frac{\mu}{2 \omega} \mathbf{n} \cdot(\mathbf{a} \times \mathbf{c})\right] } \\
& \times\left[n^{2}-\mu\left(\epsilon+\mathrm{i} \frac{\sigma}{\omega}\right)-\mathrm{i} \frac{\mu}{2 \omega} \mathbf{n} \cdot(\mathbf{a} \times \mathbf{c})\right] .
\end{aligned}
$$

In contrast to the antisymmetric magnetic conductivity of Eq. (61), the new configuration of Eq. (75) does not imply a single doubly degenerate refractive index [cf. Eq. (64)]. In contrast, we obtain two distinct refractive indices in the current scenario. Using $\mathbf{n} \cdot(\mathbf{a} \times \mathbf{c})=n|\mathbf{a}||\mathbf{c}| \cos \varphi$ in Eq. (78) results in

$$
\begin{gathered}
n_{ \pm}=\alpha_{+}^{\prime \prime}+\mathrm{i}\left(\alpha_{-}^{\prime \prime} \pm \Xi_{a, c}\right), \\
\Xi_{a, c}=\frac{\mu}{4 \omega}|\mathbf{a}||\mathbf{c}| \cos \varphi,
\end{gathered}
$$

$$
\begin{gathered}
\alpha_{ \pm}^{\prime \prime}=\sqrt{\sqrt{\Upsilon_{a, c}^{2}+\left(\frac{\mu \sigma}{2 \omega}\right)^{2}} \pm \Upsilon_{a, c}}, \\
2 \Upsilon_{a, c}=\mu \epsilon-\Xi_{a, c}^{2},
\end{gathered}
$$

with the presence of new imaginary terms stemming from the exotic conductivity and modifying the absorption coefficient. What is analogous to the antisymmetric configuration of Eq. (61) is the structure of a single one of the two refractive indices present, i.e., the dependence of the refractive index on the angle between $\mathbf{k}$ and a three-vector (b for the antisymmetric case and $\mathbf{a} \times \mathbf{c}$ for the current scenario). Also, there is again a relative minus sign between the two contributions in Eq. (79d). Therefore, we assume $\mu \epsilon \geq \Xi_{a, c}^{2}$.

A crucial difference is that two angles play a role for the current configuration: the angle $\varphi$ between $\mathbf{k}$ and $\mathbf{a} \times \mathbf{c}$ for one mode and the complementary angle $\pi-\varphi$ for the other mode. This also means that both modes interchange their roles when $\varphi$ exceeds $\pi / 2$. As the modes differ in their imaginary parts only, birefringence does not occur. It is merely the attenuation that differs for both modes.

\section{Dielectric nonconducting medium}

In this case we start from a medium with zero Ohmic conductivity $\left(\epsilon \neq 0, \sigma=0, \sigma^{B} \neq 0\right)$. Equation (79) is then reduced to 


$$
n_{ \pm}=\sqrt{2 \Upsilon_{a, c}} \pm \mathrm{i} \frac{\mu}{4 \omega}|\mathbf{a}||\mathbf{c}| \cos \varphi
$$

which exhibits an exotic absorbing behavior for nonconductive matter, with attenuation coefficient $2 \tilde{\alpha}=$ $\mu|\mathbf{a} \times \mathbf{c}| \cos \varphi$ provided $\mu \epsilon \geq \Xi_{a, c}^{2}$. In this case, the modification is proportional to $|\mathbf{a} \times \mathbf{c}|=|\mathbf{a}||\mathbf{c}|$, which is why such an effect is associated with the nondiagonal elements of $\sigma_{i j}^{B}$ instead of its trace.

Note that this nondiagonal magnetic conductivity also provides a conducting behavior for a dielectric medium, in much the same way as observed for the nondiagonal antisymmetric case [cf. Eq. (67)].

\section{Propagation modes}

In order to examine the propagating modes, we will rewrite the matrix given in Eq. (77a) for a simplified coordinate system where $\mathbf{a}=(0, a, 0)$ and $\mathbf{c}=(0,0, c)$, that is

$$
\begin{aligned}
M_{i j}= & A \delta_{i j}-n_{i} n_{j} \\
& +C\left(\epsilon_{k l j} \delta_{i 2} \delta_{k 3} n_{l}+\epsilon_{k l j} \delta_{i 3} \delta_{k 2} n_{l}\right),
\end{aligned}
$$

with

$$
A=n^{2}-\mu \epsilon, \quad C=-\mathrm{i} \frac{\mu}{2 \omega} a c .
$$

The explicit form of the matrix in Eq. (77) simplifies to

$$
\begin{aligned}
{\left[M_{i j}\right]=} & \left(\begin{array}{ccc}
A-n_{1}^{2} & -n_{1} n_{2} & -n_{1} n_{3} \\
-n_{1} n_{2} & A-n_{2}^{2} & -n_{2} n_{3} \\
-n_{1} n_{3} & -n_{2} n_{3} & A-n_{3}^{2}
\end{array}\right) \\
& +C\left(\begin{array}{ccc}
0 & 0 & 0 \\
-n_{2} & n_{1} & 0 \\
n_{3} & 0 & -n_{1}
\end{array}\right)
\end{aligned}
$$

providing the following dispersion equation:

$$
\left(A^{2}-C^{2} n_{1}^{2}\right)\left(A-n^{2}\right)=0 .
$$

Since $A-n^{2}=-\mu \epsilon$, the dispersion relations are

$$
A= \pm C n_{1},
$$

with $n_{1}=n \cos \varphi$ and corresponding to the $\sigma \mapsto 0$ limit of Eq. (78). We observe that the refractive indices depend on the direction of $\mathbf{n}$, which is defined in terms of the spherical angles $\theta$ and $\phi$ according to Eq. (42). Recalling that $\varphi$ is the angle between $\mathbf{n}$ and $\mathbf{a} \times \mathbf{c}$ we have that $\cos \varphi=\sin \theta \cos \phi$.

Taking the plus sign in Eq. (84) and using Eq. (82) we obtain

$$
E_{+}^{y}=\frac{n_{2}}{n_{1}} E_{+}^{x}, \quad E_{+}^{z}=\left(\frac{C n_{1}-n_{1}^{2}-n_{2}^{2}}{n_{1} n_{3}}\right) E_{+}^{x} .
$$

Therefore, the electric field for the plus propagating mode is

$$
\mathbf{E}_{+}=E_{+}^{(0)}\left(n_{1} n_{3}, n_{2} n_{3}, C n_{1}-n_{1}^{2}-n_{2}^{2}\right)
$$

with an appropriately chosen amplitude $E_{+}^{(0)}$. For the negative sign in the dispersion relation of Eq. (84), we obtain

$$
E_{-}^{x}=\frac{n_{1}}{n_{3}} E_{-}^{z}, \quad E_{-}^{y}=-\frac{C n_{1}+n_{1}^{2}+n_{3}^{2}}{n_{2} n_{3}} E_{-}^{z} .
$$

Thus, the electric field reads

$$
\mathbf{E}_{-}=E_{-}^{(0)}\left(n_{1} n_{2},-\left(C n_{1}+n_{1}^{2}+n_{3}^{2}\right), n_{2} n_{3}\right)
$$

with another amplitude $E_{-}^{(0)}$. We observe that $\mathbf{n} \cdot \mathbf{E}_{ \pm} \neq 0$. Equations (86) and (88) represent the propagation modes for the case of a symmetric exotic magnetic conductivity with the corresponding magnetic fields given by $\mathbf{B}_{ \pm}=\mathbf{n} \times \mathbf{E}_{ \pm}$.

\section{CONSISTENCY OF MAXWELL'S EQUATIONS}

For completeness, in this section we extend the current $\mathbf{J}$ in Eq. (2a) to include an external-source contribution $\mathbf{J}_{\mathrm{e}}$ such that now $\mathbf{J}=\mathbf{J}_{\mathrm{e}}+\sigma \cdot \mathbf{E}+\sigma^{B} \cdot \mathbf{B}$. In the previous sections, we discussed wave propagation outside of sources, which effectively meant taking $\mathbf{J}_{\mathrm{e}}=0$. Moreover, the different scenarios we have considered were defined by fixing the current $\mathbf{J}$ via specific choices of the electric and magnetic conductivities. Also, the propagation properties of the fields were obtained just by using Faraday's and Ampère's laws, incorporated into Eq. (12a), with no reference to Gauss' law given by the first of Eq. (2a).

A natural question that arises is the identification of the particular contributions to the charge density $\rho$ that are consistent with the arbitrary choice of currents, in such a way that charge conservation $\partial_{t} \rho+\nabla \cdot \mathbf{J}=0$ is preserved. To this end, we work in momentum space with the standard conventions $\nabla \mapsto \mathrm{ik}$ and $\partial_{t} \mapsto-\mathrm{i} \omega$. The main point to recognize is that Ampère's law yields

$$
\mathrm{i} \omega \mathbf{k} \cdot \mathbf{D}-\mathbf{k} \cdot \mathbf{J}=0,
$$

which together with Gauss' law $\mathrm{ik} \cdot \mathbf{D}=\rho$ gives the identification

$$
\rho=\mathbf{k} \cdot \mathbf{J} / \omega
$$

for $\omega \neq 0$, which is precisely the charge conservation condition in momentum space. In what follows, we summarize the expressions for the charge densities 
corresponding to our previous choices of the magnetic conductivity, setting $\sigma_{i j}=0$, and recalling that the Maxwell equations retain the general form of Eqs. (2a) and (2b).

For the isotropic case of Sec. III A with $\mathbf{J}=\mathbf{J}_{\mathrm{e}}+\Sigma \mathbf{B}$, the charge density is just $\rho=\rho_{\mathrm{e}}=\mathbf{k} \cdot \mathbf{J}_{\mathrm{e}} / \omega$, since $\mathbf{k} \cdot \mathbf{B}=0$. The antisymmetric case studied in Sec. III C with $\mathbf{J}=$ $\mathbf{J}_{\mathrm{e}}-\mathbf{b} \times \mathbf{B}$ yields

$$
\rho=\rho_{\mathrm{e}}-\mu \epsilon \mathbf{E} \cdot \mathbf{b}-\frac{\mathrm{i} \mu}{\omega} \mathbf{J}_{\mathrm{e}} \cdot \mathbf{b},
$$

where we used some of the Maxwell equations in order to get rid of the spatial derivatives arising from Eq. (90).

Finally, the symmetric case of Sec. III D, where we have assumed $\mathbf{a} \cdot \mathbf{c}=0$, starts from

$$
\begin{aligned}
\mathbf{J} & =\mathbf{J}_{\mathrm{e}}+\frac{1}{2}[\mathbf{a}(\mathbf{c} \cdot \mathbf{B})+\mathbf{c}(\mathbf{a} \cdot \mathbf{B})] \\
& =\mathbf{J}_{\mathrm{e}}+\mathbf{a} \times(\mathbf{c} \times \mathbf{B})-\frac{1}{2}(\mathbf{a} \times \mathbf{c}) \times \mathbf{B},
\end{aligned}
$$

and implies

$$
\begin{aligned}
\rho= & \rho_{\mathrm{e}}-\frac{\mu \epsilon}{2} \mathbf{E} \cdot(\mathbf{a} \times \mathbf{c})+\frac{1}{\omega} \mathbf{k} \cdot[\mathbf{a} \times(\mathbf{c} \times \mathbf{B})] \\
& -\frac{\mathrm{i} \mu}{2 \omega} \mathbf{J}_{e} \cdot(\mathbf{a} \times \mathbf{c}) .
\end{aligned}
$$

The alternative second form of Eq. (92) was motivated by an attempt to use the remaining Maxwell equations in favor of rewriting Eq. (90) without spatial derivatives, which, unfortunately, was not possible in this case.

\section{SOME CLASSICAL EFFECTS}

Towards the end of the paper, we intend to understand the impact that a magnetic conductivity has on certain phenomena in electrodynamics that are a consequence of material parameters such as refractive indices. As modified refractive indices for particular choices of a magnetic conductivity have already been determined earlier, we will now benefit from these findings.

\section{A. Skin depth effect}

When an electromagnetic wave falls on the surface of a conductor, its amplitude will partially penetrate the material due to the attenuation coefficient, while another part will be reflected. The characteristic penetration length into the conducting medium defines the skin depth $[30,33]$ :

$$
\bar{\delta}=\frac{1}{\omega \operatorname{Im}[n]},
$$

where $\operatorname{Im}[n]$ is the imaginary part of the complex refractive index. In the usual scenario for a simple conductor, the general skin depth reads

$$
\bar{\delta}(\omega)=\frac{1}{\omega n^{\prime \prime}}=\sqrt{\frac{2}{\mu \omega \sigma}},
$$

for a good (Ohmic) conductor and with $n^{\prime \prime}$ given by Eq. (7b). Consequently, the skin depth decreases for high frequencies.

We can now write down the skin depth for the particular symmetric (S) and antisymmetric (AS) scenarios of Sec. III C and Sec. III D, respectively, in the case when the conducting behavior is directly associated with the magnetic conductivity only, i.e., when $\sigma=0$. From Eqs. (67) and (80), we arrive at

$$
\begin{gathered}
\bar{\delta}_{A S}=\frac{2}{\mu b \cos \theta}, \\
\bar{\delta}_{S}=\frac{4}{\mu|\mathbf{a} \times \mathbf{c}| \cos \varphi} .
\end{gathered}
$$

Therefore, such a skin depth effect does not exhibit a frequency dependence, which means that the penetration length is the same for all frequency bands. This is an unusual characteristic for conductors.

\section{B. Reflection coefficient at the surface of conducting matter}

Consider a system composed of an ordinary dielectric characterized by a refractive index $n_{1}=\sqrt{\mu_{1} \epsilon_{1}}$ and a conducting phase of matter with a complex refractive index $n_{2}=n_{2}^{\prime}+\mathrm{i} n_{2}^{\prime \prime}$ described by the parameters $\epsilon_{2}, \mu_{2}$ and the Ohmic conductivity $\sigma$. For a wave that propagates from the dielectric and enters the surface of the conductor, the reflection coefficient for normal incidence is given by [30]

$$
R=\left|\frac{\mu_{1} n_{2}^{\prime}-\mu_{2} n_{1}+\mathrm{i} \mu_{1} n_{2}^{\prime \prime}}{\mu_{1} n_{2}^{\prime}+\mu_{2} n_{1}+\mathrm{i} \mu_{1} n_{2}^{\prime \prime}}\right|^{2} .
$$

Considering $n_{1} \ll n_{2}^{\prime}$ one can rewrite Eq. (97) in the general form

$$
R \approx 1-4\left(\frac{\mu_{2}}{\mu_{1}}\right) \frac{n_{1} n_{2}^{\prime}}{n_{2}^{\prime 2}+n_{2}^{\prime 2}}
$$

In standard electrodynamics, for a good conductor $\left(\sigma /\left(\omega \mu_{2}\right) \gg 1\right)$ one gets $n_{2}^{\prime}=n_{2}^{\prime \prime}=\sqrt{\mu_{2} \sigma /(2 \omega)}$. Then the reflection coefficient $R$ from Eq. (98) yields

$$
R \approx 1-2 \sqrt{2 \frac{\mu_{2} \epsilon_{1} \omega}{\mu_{1} \sigma}} .
$$

Setting $\mu_{1}=\mu_{2}$ we obtain the known Hagen-Rubens formula [30]: 


$$
R \approx 1-2 \sqrt{2 \frac{\epsilon_{1} \omega}{\sigma}} .
$$

Now we will derive the version of the latter relation for dielectric media $(\sigma=0)$ endowed with an exotic magnetic conductivity $\sigma_{i j}^{B}$.

In the scenario of an antisymmetric $\sigma_{i j}^{B}$, the refractive index is modified according to Eq. (67), so that

$n_{2}^{\prime}=\sqrt{\mu_{2} \epsilon_{2}-\left(\frac{\mu_{2}}{2 \omega} b \cos \theta\right)^{2}}, \quad n_{2}^{\prime \prime}=\frac{\mu_{2}}{2 \omega} b \cos \theta$.

By inserting Eq. (101) into Eq. (98), one obtains

$$
R_{A S} \approx 1-4 \sqrt{\frac{\mu_{2} \epsilon_{1}}{\mu_{1} \epsilon_{2}}} \sqrt{1-\frac{\mu_{2}}{\epsilon_{2}}\left(\frac{b \cos \theta}{2 \omega}\right)^{2}},
$$

for real $n_{2}^{\prime}$. This result is a Hagen-Rubens-like formula for the case when there is a contribution from the antisymmetric magnetic conductivity only. It is also very different from the reflection coefficient for an ordinary dielectric,

$$
R \approx 1-4 \sqrt{\frac{\mu_{2} \epsilon_{1}}{\mu_{1} \epsilon_{2}}}
$$

since the magnetic conductivity introduces a frequencydependent term in $R$. Hence, the exotic conductivity results in a conducting-matter phase in the limit $\sigma \mapsto 0$.

In the scenario of a symmetric $\sigma_{i j}^{B}$, one obtains a similar result:

$$
R_{S} \approx 1-4 \sqrt{\frac{\mu_{2} \epsilon_{1}}{\mu_{1} \epsilon_{2}}} \sqrt{1-\frac{\mu_{2}}{\epsilon_{2}}\left(\frac{|\mathbf{a}||\mathbf{c}| \cos \varphi}{4 \omega}\right)^{2}} .
$$

\section{FINAL REMARKS}

Electrodynamics in matter is well described by the Maxwell equations and the constitutive relations. Extensions of the Maxwell equations including the possibility of a chiral magnetic current is a topical issue [14-21]. In this work, we have extended the scenario of electric currents generated by magnetic fields by studying some basic classical properties of a magnetic conductivity implemented into the Maxwell equations through the extension of Ohm's law given in Eq. (11).

The main purpose was to examine the propagation of electromagnetic waves in dispersive dielectric materials, paying attention to the conduction properties induced upon non-Ohmic materials $(\sigma=0)$. To this end, we proposed some particular realizations for the magnetic-conductivity tensor $\sigma_{i j}^{B}$ : (i) a diagonal isotropic and a diagonal anisotropic tensor (which include the chiral magnetic effect) in Secs. III A and III B (ii) a nondiagonal antisymmetric tensor in Sec. III C, and (iii) a traceless nondiagonal symmetric tensor in Sec. III D. Let us point out that the trace of the magnetic-conductivity tensor $\sigma_{i j}^{B}$ is related to the chiral magnetic effect and that the off-diagonal components of $\sigma_{i j}^{B}$ describe generalizations of such an effect. All these configurations induce parity violation, since the associated current entering Ampère's law is linear in $\mathbf{B}$.

We have verified that a diagonal isotropic tensor $\sigma_{i j}^{B}=$ $\Sigma \delta_{i j}$ modifies the refractive index of a dispersive dielectric medium yielding two distinct complex values $n_{ \pm}$. Most notably, these results are independent of the propagation direction, implying what we could call an "isotropic birefringence." In the $\sigma=0$ case, the resulting refractive indices are real and the propagation modes correspond to left- and right-handed circular polarizations. As such, these media can be characterized as optically active having a frequencyindependent specific rotatory power $\Delta=-\mu \Sigma / 2$. This is a consequence of the EPT being Hermitian and the electric field being orthogonal to the wave vector $\mathbf{k}$. In this case, parity violation manifests itself only in the fact that $n_{+}$is different from $n_{-}$.

The diagonal anisotropic case also exhibits birefringence, but this time the complex refractive indices are direction dependent. Nevertheless, the functions depend on the squares of the momentum components $k_{i}$ for $i=1,2,3$ and are insensitive to parity transformations. Thus, the violation of this symmetry is again manifest only in the different values of $n_{ \pm}$. The EPT is non-Hermitian and $\mathbf{k} \cdot \mathbf{E} \neq 0$, in general, thus preventing the description of polarization in terms of left- and right-handed modes.

Focusing on the $\sigma=0$ case, we find again real refractive indices. In this situation we have explored in detail the case of propagation along $\mathbf{n}=\left(n_{1}, n_{2}, 0\right)$ showing explicitly that the eigenvectors of the EPT do not correspond to the polarization modes of the electric field. A further particular case of propagation along $\mathbf{n}=\left(0, n_{2}, 0\right)$ restores the orthogonality between $\mathbf{k}$ and $\mathbf{E}$ and ends up with elliptically polarized propagation modes. The case of circular polarization is recovered when taking the isotropic limit $\Sigma_{i}=\Sigma$, which also reproduces the previous expressions for the refractive indices. As expected, in this specific situation the eigenvectors of the EPT and the propagation modes coincide. We remark that both cases of a diagonal chiral conductivity tensor do not induce a conducting behavior in a non-Ohmic dielectric medium.

We have also examined a nondiagonal (antisymmetric and symmetric) $\sigma_{i j}^{B}$. In these cases, the off-diagonal components of $\sigma_{i j}^{B}$ provide complex refractive indices even for a vanishing Ohmic conductivity $(\sigma=0)$, which leads to the remarkable behavior of a conducting phase in the dielectric substrate. The magnetic conductivity in these cases implies nonzero absorption coefficients that damp the intensity of electromagnetic waves propagating through the medium. In both cases, the EPT is non-Hermitian, $\mathbf{k} \cdot \mathbf{E} \neq 0$, and the refractive indices are direction 
dependent. The latter exhibit contributions that obviously induce parity violation.

A rather unexpected feature in the context of a parityviolating theory occurs for the antisymmetric case, which is the absence of birefringence. That is to say, we obtain only one doubly degenerate value for the refractive index, which nevertheless supports two orthogonal polarization modes, as shown in general when $\sigma=0$. On the contrary, the symmetric case exhibits birefringence.

For the nondiagonal cases (antisymmetric or symmetric) we have calculated the associated skin depth, which in the limit $\sigma \mapsto 0$ becomes a constant for all frequency bands of the electromagnetic wave that enters the medium. We also have derived a generalization of the Hagen-Rubens relation for nonconducting media $(\sigma=0)$, with contributions stemming from the magnetic conductivity only. In this case, the reflection coefficient is frequency dependent, which is a property that does not occur at the interface of two ordinary dielectric substrates.

It is interesting to observe that when $\sigma=0$, the equation determining the refractive indices in all four cases considered boils down to the form

$$
n^{2}-\mu \epsilon=n B
$$

where $B$ specifies each case according to

$$
B= \pm \frac{\mu \Sigma}{\omega},
$$

for the isotropic diagonal case of Sec. III A,

$$
B= \pm \frac{\sqrt{\tilde{\Omega}}}{\omega},
$$

for the anisotropic diagonal case of Sec. III B,

$$
B=\mathrm{i} \frac{\mu}{\omega} b \cos \theta
$$

for the antisymmetric case of Sec. III C and finally,

$$
B= \pm \mathrm{i} \frac{\mu}{2 \omega}|\mathbf{a}||\mathbf{c}| \cos \varphi
$$

for the symmetric case of Sec. III D.

A comment in relation to the consistency of our calculation is now in order. A current density $\mathbf{J}$ linear in the magnetic field has been the only additional input we have introduced into the calculation of the propagation properties of a wave, without making any statement about the corresponding charge density required by current conservation. The point to be recalled is that when $\omega \neq 0$, Ampère's law directly yields charge conservation via the use of Gauss' law, as shown in Sec. IV. Thus, for completeness, in the latter section we have identified the charge densities corresponding to some of the currents we have introduced before. Perhaps an unexpected feature is that some of the parameters defining such currents (b and $\mathbf{a} \times \mathbf{c}$, for example) give rise to additional contributions to the charge density proportional to the external currents that we have set equal to zero in this analysis.

Finally, we conclude that this work presents a classical perspective of some novel effects that a magnetic conductivity can provide for the propagation of electromagnetic waves in dispersive media.

\section{ACKNOWLEDGMENTS}

The authors thank S. Kaushik, D. E. Kharzeev, and E. J. Philip for valuable discussions as to their work [36] as well as M. Kaminski and C. Valagiannopoulos for additional comments. P. D. S. S., M. M. F., and M. S. express their gratitude to FAPEMA, CNPq and CAPES (Brazilian research agencies) for invaluable financial support. In particular, M. M. F. is supported by FAPEMA Universal 01187/18, and CNPq Produtividade 311220/2019-3. M. S. receives support from FAPEMA Universal 01149/17, CNPq Universal 421566/2016-7, and CNPq Produtividade 312201/2018-4. Furthermore, we are indebted to CAPES/Finance Code 001. L. F. U. acknowledges support from the project DGAPAUNAM-IN103319.
[1] D. E. Kharzeev, The chiral magnetic effect and anomalyinduced transport, Prog. Part. Nucl. Phys. 75, 133 (2014); D. E. Kharzeev, J. Liao, S. A. Voloshin, and G. Wang, Chiral magnetic and vortical effects in high-energy nuclear collisions-A status report, Prog. Part. Nucl. Phys. 88, 1 (2016); D. Kharzeev, K. Landsteiner, A. Schmitt, and H. U. Yee, Strongly Interacting Matter in Magnetic Fields, Lect. Notes Phys. Vol. 871 (Springer-Verlag, Berlin, Heidelberg, 2013).
[2] K. Fukushima, D. E. Kharzeev, and H. J. Warringa, Chiral magnetic effect, Phys. Rev. D 78, 074033 (2008).

[3] G. Inghirami, M. Mace, Y. Hirono, L. Del Zanna, D. E. Kharzeev, and M. Bleicher, Magnetic fields in heavy ion collisions: flow and charge transport, Eur. Phys. J. C 80, 293 (2020).

[4] J. Schober, A. Brandenburg, and I. Rogachevskii, Chiral fermion asymmetry in high-energy plasma simulations, Geophys. Astrophys. Fluid Dyn. 114, 106 (2020). 
[5] A. Vilenkin, Equilibrium parity-violating current in a magnetic field, Phys. Rev. D 22, 3080 (1980); A. Vilenkin and D. A. Leahy, Parity nonconservation and the origin of cosmic magnetic fields, Astrophys. J. 254, 77 (1982).

[6] M. Dvornikov and V. B. Semikoz, Influence of the turbulent motion on the chiral magnetic effect in the early universe, Phys. Rev. D 95, 043538 (2017).

[7] G. Sigl and N. Leite, Chiral magnetic effect in protoneutron stars and magnetic field spectral evolution, J. Cosmol. Astropart. Phys. 01 (2016) 025.

[8] M. Dvornikov and V. B. Semikoz, Magnetic field instability in a neutron star driven by the electroweak electron-nucleon interaction versus the chiral magnetic effect, Phys. Rev. D 91, 061301(R) (2015).

[9] A. F. Bubnov, N. V. Gubina, and V. Ch. Zhukovsky, Vacuum current induced by an axial-vector condensate and electron anomalous magnetic moment in a magnetic field, Phys. Rev. D 96, 016011 (2017).

[10] Y. Akamatsu and N. Yamamoto, Chiral Plasma Instabilities, Phys. Rev. Lett. 111, 052002 (2013); A. Boyarsky, O. Ruchayskiy, and M. Shaposhnikov, Long-Range Magnetic Fields in the Ground State of the Standard Model Plasma, Phys. Rev. Lett. 109, 111602 (2012).

[11] M. Dvornikov and V. B. Semikoz, Instability of magnetic fields in electroweak plasma driven by neutrino asymmetries, J. Cosmol. Astropart. Phys. 05 (2014) 002; M. Dvornikov, Chiral magnetic effect in the presence of an external axial-vector field, Phys. Rev. D 98, 036016 (2018).

[12] M. Dvornikov, Electric current induced by an external magnetic field in the presence of electroweak matter, EPJ Web Conf. 191, 05008 (2018).

[13] A. A. Burkov, Chiral anomaly and transport in Weyl metals, J. Phys. Condens. Matter 27, 113201 (2015).

[14] Q. Li, D. E. Kharzeev, C. Zhang, Y. Huang, I. Pletikosić, A. V. Fedorov, R. D. Zhong, J. A. Schneeloch, G. D. Gu, and T. Valla, Chiral magnetic effect in $\mathrm{ZrTe}_{5}$, Nat. Phys. 12, 550 (2016).

[15] M.-C. Chang and M.-F. Yang, Chiral magnetic effect in a two-band lattice model of Weyl semimetal, Phys. Rev. B 91, 115203 (2015).

[16] E. C. I van der Wurff and H. T. C. Stoof, Anisotropic chiral magnetic effect from tilted Weyl cones, Phys. Rev. B 96, 121116(R) (2017).

[17] K. Landsteiner, Anomalous transport of Weyl fermions in Weyl semimetals, Phys. Rev. B 89, 075124 (2014).

[18] S. Kaushik and D. E. Kharzeev, Quantum oscillations in the chiral magnetic conductivity, Phys. Rev. B 95, 235136 (2017).

[19] A. Martín-Ruiz, M. Cambiaso, and L. F. Urrutia, Electromagnetic fields induced by an electric charge near a Weyl semimetal, Phys. Rev. B 99, 155142 (2019).

[20] D. E. Kharzeev and H. J. Warringa, Chiral magnetic conductivity, Phys. Rev. D 80, 034028 (2009); D. E. Kharzeev, Chiral magnetic superconductivity, EPJ Web Conf. 137, 01011 (2017).

[21] Z. Qiu, G. Cao, and X.-G. Huang, Electrodynamics of chiral matter, Phys. Rev. D 95, 036002 (2017).

[22] V. A. Kostelecký and S. Samuel, Spontaneous breaking of Lorentz symmetry in string theory, Phys. Rev. D 39, 683
(1989); V. A. Kostelecký and R. Potting, CPT and strings, Nucl. Phys. B 359, 545 (1991); CPT, strings, and meson factories, Phys. Rev. D 51, 3923 (1995).

[23] D. Colladay and V. A. Kostelecký, CPT violation and the standard model, Phys. Rev. D 55, 6760 (1997); D. Colladay and V. A. Kostelecký, Lorentz-violating extension of the standard model, Phys. Rev. D 58, 116002 (1998); S. Coleman and S. L. Glashow, High-energy tests of Lorentz invariance, Phys. Rev. D 59, 116008 (1999).

[24] S. M. Carroll, G. B. Field, and R. Jackiw, Limits on a Lorentz- and parity-violating modification of electrodynamics, Phys. Rev. D 41, 1231 (1990); A. A. Andrianov and R. Soldati, Lorentz symmetry breaking in Abelian vector-field models with Wess-Zumino interaction, Phys. Rev. D 51, 5961 (1995); A. A. Andrianov and R. Soldati, Patterns of Lorentz symmetry breaking in QED by $C P T$-odd interaction, Phys. Lett. B 435, 449 (1998); A. A. Andrianov, R. Soldati, and L. Sorbo, Dynamical Lorentz symmetry breaking from a $(3+1)$-dimensional axion-Wess-Zumino model, Phys. Rev. D 59, 025002 (1998); J. Alfaro, A. A. Andrianov, M. Cambiaso, P. Giacconi, and R. Soldati, Bare and induced Lorentz and CPT invariance violations in QED, Int. J. Mod. Phys. A 25, 3271 (2010); A. A. Andrianov, D. Espriu, P. Giacconi, and R. Soldati, Anomalous positron excess from Lorentz-violating QED, J. High Energy Phys. 09 (2009) 057.

[25] V. A. Kostelecký and M. Mewes, Cosmological Constraints on Lorentz Violation in Electrodynamics, Phys. Rev. Lett. 87, 251304 (2001); Signals for Lorentz violation in electrodynamics, Phys. Rev. D 66, 056005 (2002); Sensitive Polarimetric Search for Relativity Violations in GammaRay Bursts, Phys. Rev. Lett. 97, 140401 (2006); C. A. Escobar and M. A. G. Garcia, Full CPT-even photon sector of the standard model extension at finite temperature, Phys. Rev. D 92, 025034 (2015); A. Martín-Ruiz and C. A. Escobar, Casimir effect between ponderable media as modeled by the standard model extension, Phys. Rev. D 94, 076010 (2016).

[26] Q. G. Bailey and V.A. Kostelecký, Lorentz-violating electrostatics and magnetostatics, Phys. Rev. D 70, 076006 (2004).

[27] D. A. Gurnett and A. Bhattacharjee, Introduction to Plasma Physics (Cambridge University Press, Cambridge, England, 2005).

[28] J. F. Nieves and P. B. Pal, Third electromagnetic constant of an isotropic medium, Am. J. Phys. 62, 207 (1994).

[29] A. Martín-Ruiz, M. Cambiaso, and L. F. Urrutia, Electroand magnetostatics of topological insulators as modeled by planar, spherical, and cylindrical $\theta$ boundaries: Green's function approach, Phys. Rev. D 93, 045022 (2016).

[30] A. Zangwill, Modern Electrodynamics (Cambridge University Press, New York, 2012).

[31] R. S. Kshetrimayum, A brief intro to metamaterials, IEEE Potentials 23, 44 (2004).

[32] L. D. Landau and E. M. Lifshitz, Electrodynamics of Continuous Media, Course of Theoretical Physics Vol. 8, 2nd ed. (Pergamon Press, New York, 1984).

[33] J. D. Jackson, Classical Electrodynamics, 3rd ed. (John Wiley \& Sons, New York, 1999). 
[34] L. A. Pajdzik and A. M. Glazer, Three-dimensional birefringence imaging with a microscope tilting-stage. I. Uniaxial crystals, J. Appl. Crystallogr. 39, 326 (2006).

[35] I. G. Wood and A. M. Glazer, Ferroelastic phase transition in $\mathrm{BiVO}_{4}$. I. Birefringence measurements using the rotatinganalyser method, J. Appl. Crystallogr. 13, 217 (1980);
M. A. Geday, W. Kaminsky, J. G. Lewis, and A. M. Glazer, Images of absolute retardance $L \cdot \Delta n$, using the rotating polariser method, J. Microsc. 198, 1 (2000).

[36] S. Kaushik, D. E. Kharzeev, and E. J. Philip, Transverse chiral magnetic photocurrent induced by linearly polarized light in symmetric Weyl semimetals, arXiv:2006.04857. 